\title{
Developments of the method of difference potentials for linear elastic fracture mechanics problems
}

DOI:

10.1002/nme.5796

Document Version

Accepted author manuscript

Link to publication record in Manchester Research Explorer

\section{Citation for published version (APA):}

Woodward, H., Utyuzhnikov, S., \& Massin, P. (2018). Developments of the method of difference potentials for linear elastic fracture mechanics problems. International Journal for Numerical Methods in Engineering. https://doi.org/10.1002/nme.5796

\section{Published in:}

International Journal for Numerical Methods in Engineering

\section{Citing this paper}

Please note that where the full-text provided on Manchester Research Explorer is the Author Accepted Manuscript or Proof version this may differ from the final Published version. If citing, it is advised that you check and use the publisher's definitive version.

\section{General rights}

Copyright and moral rights for the publications made accessible in the Research Explorer are retained by the authors and/or other copyright owners and it is a condition of accessing publications that users recognise and abide by the legal requirements associated with these rights.

\section{Takedown policy}

If you believe that this document breaches copyright please refer to the University of Manchester's Takedown Procedures [http://man.ac.uk/04Y6Bo] or contact uml.scholarlycommunications@manchester.ac.uk providing relevant details, so we can investigate your claim.

\section{OPEN ACCESS}




\title{
Developments of the Method of Difference Potentials for Linear Elastic Fracture Mechanics Problems
}

\author{
W. H. Woodward ${ }^{1 * \dagger}$, S. Utyuzhnikov ${ }^{2,3}$ and P. Massin ${ }^{4}$ \\ ${ }^{1}$ Centre of Environmental Policy, Imperial College London, SW7 1NA, UK \\ ${ }^{2}$ School of Mechanical, Aerospace and Civil Engrg., Univ. of Manchester, M13 9PL, UK \\ ${ }^{3}$ Moscow Institute of Physics and Technology, Dolgoprundy, Russia \\ ${ }^{4}$ Institut des Sciences de la Mécanique et Applications Industrielles, UMR EDF-CNRS-CEA-ENSTA ParisTech, \\ Université Paris-Saclay, 828 Boulevard des Marchaux, 91120, Palaiseau
}

\begin{abstract}
SUMMARY
Recently the Method of Difference Potentials has been extended to Linear Elastic Fracture Mechanics. The solution was calculated on a grid boundary belonging to the domain of an auxiliary problem, which must be solved multiple times. Singular enrichment functions, such as those used within the Extended Finite Element Method, were introduced in order to improve the approximation near the crack tip leading to near-optimal convergence rates. Now the method is further developed by significantly reducing the computation time. This is achieved via the implementation of a system of basis functions introduced along the physical boundary of the problem. The basis functions form an approximation of the trace of the solution at the physical boundary. This method has proven efficient for the solution of problems on regular (Lipschitz) domains. By introducing the singularity into the finite element space, the approximation of the crack can be realised by regular functions. Near optimal convergence rates are then achieved for the enriched formulation. A solution algorithm using the Fast Fourier Transform is provided with the aim of further increasing the efficiency of the method.
\end{abstract}

Copyright (c) 2010 John Wiley \& Sons, Ltd.

Received ...

KEY WORDS: method of difference potentials; extended finite element method; fracture; rate of convergence; fast Fourier transform

\section{INTRODUCTION}

The Method of Difference Potentials, (DPM), was originally developed by Ryaben'kii [1,2] and has been applied to Boundary Value Problems (BVPs) in fluid dynamics [3], acoustics [4, 5, 6] and many other applications [1]. The methodology is based on the same boundary integral equation as that used in Boundary Element Methods (BEM) [7, 8, 9, 10, 11]. Within the BEM, this boundary integral equation is numerically integrated given the knowledge of a suitable Green's function. The DPM

\footnotetext{
${ }^{*}$ Correspondence to: W. H. Woodward, Centre of Environmental Policy, Imperial College London, SW7 1NA, UK.

†E-mail: huw.woodward@imperial.ac.uk 
avoids any need for Green's functions by embedding the problem within a well-defined auxiliary problem. The auxiliary problem is then solved numerically, avoiding the solution of the integral equation. A boundary system of equations must be constructed in order to calculate appropriate grid densities at the embedded boundary. This requires the auxiliary problem to be solved a multiple number of times, where the number of solutions required increases with required accuracy. Definition of the auxiliary problem can be flexible provided that it is well defined and completely encloses the embedded domain and its boundary. The auxiliary problem is therefore chosen as to facilitate the most efficient solution method. Previously a square domain with a regular grid has been used along with the use of finite difference methods which can be particularly efficient for regular problems such as this. In this paper, an adjusted Extended Finite Element Method (XFEM) formulation based on that used in [12] is used to solve the auxiliary problem. Unlike other embedded methods, such as the Finite Cell Method [13], the DPM does not require any special quadrature procedure near the boundary in order to accurately represent the domain geometry. The adjusted XFEM used here extends the near-crack-tip singular enrichment functions along the entire length of the crack, replacing the traditional Heaviside enrichment. While this adjustment is reasonable for the simple and well understood test cases solved in this paper, it is recognized that the traditional combination of Heaviside and singular enrichment is required for general problems. However, for the purpose of demonstrating the promise of this method for further development, the adjusted XFEM is used for simplicity of formulation.

Recently the DPM was applied to Linear Elastic Fracture Mechanics (LEFM) [12]. For the first time, DPM technology was combined with the finite element method (FEM). By introducing singular enrichment functions near the crack tip, such as those used in XFEM, it was shown that the DPM can achieve near-optimal convergence rates for simple 2D crack test cases. In addition to this, the minimal clear trace for crack problems, as derived by [14], was numerically realised. Several developments of the methodology given in [12] for the solution of LEFM problems are presented here. In [12] the solution to the problem was based on a grid boundary close to the physical boundary of the problem. A system of basis functions is now introduced along the physical boundary, as suggested in [1] for regular problems. The basis functions form an approximation of the trace of the solution at the physical boundary. This method has proven efficient for the solution of problems on regular (Lipschitz) domains, e.g. [15]. So far there have only been a few examples where the DPM has been used for solving BVPs with singularities. For the first time the DPM was applied in non-Lipschitz domains for the solution of the Poisson and Chaplygin equations in [16, 17, 18]. In [19] the Helmholtz equation is solved for a problem with a singularity which exists due to a nonsmooth boundary. In addition, work [20] is worth noting in which a discontinuity in the boundary conditions is considered. In these papers the regularization of the numerical algorithm was based on the identification of an explicit asymptotic behaviour of the solution near the singularity.

In the current work the FEM basis is enriched near the crack tip in order to account for the singularity. Local splines are used for the basis functions to localise any error anticipated near the singularity. However it is found that by enriching the FEM space with the appropriate singular functions, the trace along the crack boundary can be reduced to a regular function. Therefore, no special treatment is required along the crack boundary despite the presence of a discontinuity across the crack and a singularity at the crack tip. Competitive convergence rates are achieved. The reduction of the problem to the physical boundary allows for a significant reduction of the number of 
required solutions of the auxiliary problem. Therefore the method is significantly faster than when solving on the grid boundary, as in [12]. Further to this, by interpolating from the grid boundary to the physical boundary, boundary conditions of all kinds can be applied more easily than for the previous method.

Although multiple solutions of the auxiliary problem are still required, the DPM's ability to solve complex problems on a regular grid leads to advantages prior to solving the problem. Firstly, there is no need to generate a mesh which conforms to the domain geometry. Secondly, the stiffness matrix can be defined explicitly using a finite difference scheme, thus avoiding the expensive Gauss quadrature procedure required for the FEM formulation. This includes avoiding any special quadrature procedures needed near the crack tip. Therefore two expensive preprocessing steps can be avoided by the method. In order to further increase the efficiency of the method, we suggest a Fast Fourier Transform (FFT) algorithm for the fast solution of the auxiliary problem. The algorithm is based on that given by Wiegmann [21] for the solution of two-dimensional finite difference equations. Here it is given for a FEM formulation and a two-stage solution is required in order to account for the enriched space. The method takes advantage of the regular grid used in order to solve the problem using the FFT. A coupled scheme is used where the relatively small enriched part is solved using conventional methods while the large, regular part is solved using the FFT. This leads to a fast solution method for the enriched system when the enriched area is small relative to the total area of the domain. It also holds the advantage of avoiding the need to construct a full stiffness matrix for the problem. Here the formulation is applied to a square auxiliary domain, however it remains valid for rectangular domains provided that the grid is regular. A first discussion is given of the efficiency of this formulation and further numerical tests will be presented in a future paper.

This paper leads directly from the work presented in [12]. The reader is therefore encouraged to first familiarise themselves with the work presented there. This paper is organised as follows. Section 2 defines the problem to be solved along with the auxiliary problem used within the DPM. Section 3 outlines the XFEM formulation used to solve the auxiliary problem. The clear trace used at the crack boundary is described in Section 4.1 while an approximation of the differential potentials and projections are formulated in Section 5. A numerical algorithm for the enriched DPM is provided in Section 6. The result of convergence studies for simple 2D test cases are given in Section 7. In Section 8 a fast solution algorithm using the FFT is outlined. A brief discussion of the method and the findings of this paper are provided in Section 9 and a conclusion is given in Section 10 .

\section{DEFINITION OF THE PROBLEM}

\subsection{Crack problem}

A linear elastic bidimensional problem is considered for an isotropic homogeneous material. The problem is considered on a bounded domain $\bar{D} \in \mathbb{R}^{2}$ with outer boundary $\Gamma^{\prime}$ and containing a crack along a contour denoted $\Gamma_{c}=\Gamma_{c_{l}} \cup \Gamma_{c_{r}}$, where $\Gamma_{c_{l}}$ and $\Gamma_{c_{r}}$ denote the left and right-hand side of the crack boundary respectively. We denote $\Gamma=\Gamma^{\prime} \cup \Gamma_{c}$ and therefore we have $\bar{D}=D \bigcup \Gamma$, as seen in Fig. 1(a). The displacements over the domain are given as the vector functions $v_{\bar{D}}=$ $\left\{v_{\bar{D}}^{(1)}, v_{\bar{D}}^{(2)}\right\} \in C^{2}\left(D \backslash \Gamma_{c}\right) \bigcap C^{1}\left(\bar{D} \backslash \Gamma_{c}\right)$ where $C^{k}$ denotes the space of functions for which their 
first $k$ derivatives are smooth and continuous and the superscripts (1) and (2) denote the $x$ and $y$ components respectively. $v_{\bar{D}}$ can be discontinuous at the crack boundary $\Gamma_{c}$.

The displacement functions satisfy the two-dimensional elastostatic system:

$$
\mu \Delta v_{\bar{D}}+(\lambda+\mu) \operatorname{grad} \operatorname{div} v_{\bar{D}}=f_{D}
$$

where $\lambda>0$ is the Lame constant and $\mu>0$ is the shear modulus of the material. The right-hand side, $f_{D}=\left\{f_{D}^{(1)}, f_{D}^{(2)}\right\}$, represents body forces acting upon the domain and we define $V_{\bar{D}}$ as the space of all admissible displacement functions $v_{\bar{D}}$. The BVP to be solved is defined in operator form as:

$$
\begin{array}{cl}
\mathbf{L}_{D \bar{D}} v_{\bar{D}}=f_{D} & \text { on } \bar{D}, \\
\mathbf{l}_{\Gamma \bar{D}} v_{\bar{D}}=\varphi_{\Gamma} & \text { on } \Gamma,
\end{array}
$$

where $\mathbf{L}_{D \bar{D}}$ and $\mathbf{l}_{\Gamma \bar{D}}$ are linear operators representing the elastostatic equation (1) and boundary conditions respectively. We can then say that $f_{D}$ belongs to the space of functions $F_{D}$ such that $\mathbf{L}_{D \bar{D}}: V_{\bar{D}} \rightarrow F_{D}$. The boundary operator, $1_{\Gamma \bar{D}}$, can define Dirichlet, Neumann or mixed boundary conditions and $\varphi_{\Gamma}$ is a known boundary function. We assume that $f_{D}$ and $\varphi_{\Gamma}$ are such that the BVP (2) is well posed if $v_{\bar{D}} \in C^{2}\left(D \backslash \Gamma_{c}\right) \bigcap C^{1}\left(\bar{D} \backslash \Gamma_{c}\right)$. We also introduce the subspace $U_{\bar{D}} \subset V_{\bar{D}}$ of all regular displacement functions which are smooth and continuous across the crack such that in this case effectively no crack boundary exists.

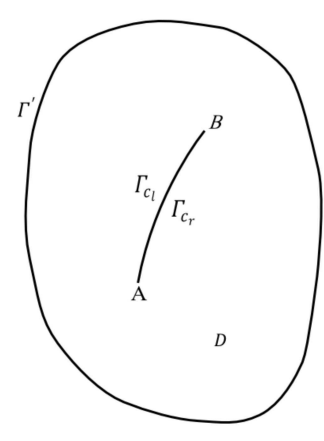

(a)

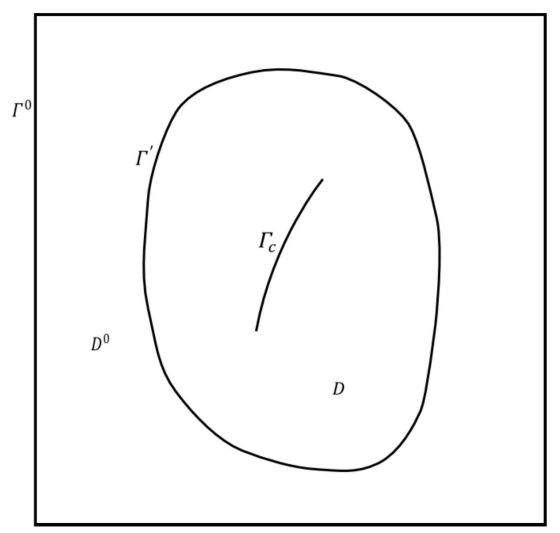

(b)

Figure 1. (a) Cracked domain $\bar{D}=D \bigcup \Gamma$ with $\Gamma=\Gamma^{\prime} \bigcup \Gamma_{c}$ and (b) the auxiliary domain $\bar{D}^{0}=D^{0} \cup \Gamma^{0}$

Let us introduce the Mode I and Mode II asymptotic displacement fields. Let $r$ denote the distance from the crack tip and $\theta \in[-\pi ; \pi]$ be the angle to the tangential of the crack at the crack tip. The displacements fields are then given as:

$$
\begin{aligned}
& v_{I}^{(1)}=\frac{K_{I}}{2 \mu} \sqrt{\frac{r}{2 \pi}} \cos \left(\frac{\theta}{2}\right)\left[\kappa-1+2 \sin ^{2}\left(\frac{\theta}{2}\right)\right], \\
& v_{I}^{(2)}=\frac{K_{I}}{2 \mu} \sqrt{\frac{r}{2 \pi}} \sin \left(\frac{\theta}{2}\right)\left[\kappa+1-2 \cos ^{2}\left(\frac{\theta}{2}\right)\right],
\end{aligned}
$$




$$
\begin{gathered}
v_{I I}^{(1)}=\frac{K_{I I}}{2 \mu} \sqrt{\frac{r}{2 \pi}} \sin \left(\frac{\theta}{2}\right)\left[\kappa+1+2 \cos ^{2}\left(\frac{\theta}{2}\right)\right], \\
v_{I I}^{(2)}=-\frac{K_{I I}}{2 \mu} \sqrt{\frac{r}{2 \pi}} \cos \left(\frac{\theta}{2}\right)\left[\kappa-1-2 \sin ^{2}\left(\frac{\theta}{2}\right)\right] .
\end{gathered}
$$

Here $K_{I}$ and $K_{I I}$ are the Mode I and Mode II Stress Intensity Factors (SIFs) respectively. $\kappa$ is Kosolov's constant and is equal to $(3-4 \nu)$ for plane strain and $(3-\nu) /(1+\nu)$ for plane stress, where $\nu$ is Poisson's ratio. These displacement fields will be used to define the crack test cases to be solved in this paper (Section 7.1) in addition to the enrichment functions used within the XFEM formulation (Section 3.2). It is to be noted that although the asymptotic form of the solution near the singularity is assumed to be known, the corresponding coefficients, or the appropriate weights, are not known. They are to be included into the overall set of unknowns and determined in the numerical solution. It is also noted that only the first terms of the asymptotic solution are given by 3 and 4 . The higher order terms have been truncated as they are not required for the problems considered in this paper with low order boundary conditions.

Higher order terms are required if higher order basis functions are to be used [22]. It has also been found that including higher order terms can significantly improve the accuracy of the stress near the crack tip [23]. This is particularly useful for dynamic crack propagation models as it provides a quick method to calculate the stress intensity factors which in turn allows the direction of propagation to be calculated. Introducing higher order terms involves introducing additional coefficients within the enriched area near the crack. This should not introduce a significant complication to the formulation other than causing the stiffness matrix to be poorly conditioned. For higher order terms it may be the case that a preconditioner would be needed for the stiffness matrix.

\subsection{Auxiliary problem}

The BVP to be solved is embedded within an auxiliary domain of simple geometry discretized using a uniform grid. This section describes the construction of the auxiliary problem used in the solution process of the DPM.

For a domain $\bar{D}$ which lies within an auxiliary domain $D^{0}$ bounded by $\Gamma^{0}$ so that $\bar{D} \subset D^{0}$, as shown in Fig. 1(b), let us define a function $v_{\bar{D}}$ which extends over the entirety of $\bar{D}^{0}$ such that $v_{\bar{D}}=\left.v_{\bar{D}^{0}}\right|_{\bar{D}}$. The auxiliary domain is defined as a square $D^{0}=\{x, y \mid 0 \leq x \leq L, 0 \leq y \leq L\}$ where $L$ is the length of the domain. We define the space of all admissible displacements within $\bar{D}^{0}$ as $V_{\bar{D}^{0}} \subset C^{2}\left(D^{0} \backslash \Gamma_{c}\right) \bigcap C^{1}\left(\bar{D}^{0} \backslash \Gamma_{c}\right)$ where all $v_{\bar{D}^{0}} \in V_{\bar{D}^{0}}$ satisfy the homogeneous boundary conditions defined by $\mathrm{l}_{\Gamma^{0} \bar{D}^{0}} v_{\bar{D}^{0}}=0$. We note that $\Gamma^{0} \bigcap \Gamma_{c}=\emptyset$ and therefore here there is no boundary condition applied to the functions $v_{\bar{D}^{0}}$ on the crack boundary $\Gamma_{c}$. Let $U_{\bar{D}^{0}}$ be the space of all regular functions $u_{\bar{D}^{0}}$ which are smooth and continuous across the crack (or equivalently, for which no crack boundary exists, i.e. $\Gamma_{c}=\emptyset$ ), such that $U_{\bar{D}^{0}} \subset V_{\bar{D}^{0}}$.

Let $F_{D^{0}}$ be the space of all body force functions $f_{D^{0}}$ where $\operatorname{supp} f_{D^{0}}=D$, i.e. $f_{D^{0}}(x)=0$ if $x \notin D$. The boundary condition defined by $\mathrm{l}_{\Gamma^{0} \bar{D}^{0}}$ must ensure that the solution to the regular problem is well-posed. However beyond this it can be chosen as to facilitate the most efficient numerical solution of the problem. For most of this paper we shall use uniform Dirichlet boundary conditions along the auxiliary boundary, $\Gamma^{0}$, in the form $\left.u_{\bar{D}^{0}}\right|_{\Gamma^{0}}=0$ for all $u_{\bar{D}^{0}} \in U_{\bar{D}^{0}}$, until Section 
8 where we use mixed boundary conditions. The auxiliary problem with no crack is therefore defined as:

$$
\mathbf{L}_{D^{0} \bar{D}^{0}} u_{\bar{D}^{0}}=f_{D^{0}}, \quad u_{\bar{D}^{0}} \in U_{\bar{D}^{0}}, \quad f_{D^{0}} \in F_{D^{0}} .
$$

This auxiliary problem will be used to find the solution of the embedded BVP (2) for which a solution is required. The DPM for domains containing a crack requires the solution of this regular auxiliary problem (5) only.

\section{METHOD USED TO SOLVE AUXILIARY PROBLEM}

An XFEM formulation is used in order to model crack problems. This is done by introducing a discontinuity across the crack, in addition to an approximation of the singularity at the crack tip, into the solution space. Although the DPM only requires multiple solutions of a regular auxiliary problem, a discontinuous auxiliary problem must be formulated in order to calculate the appropriate force terms for the regular auxiliary problems to be solved. However a solution is never required for the discontinuous problem. The adjusted XFEM used here is based on the XFEM formulation presented in [12].

\subsection{Definition of grid sets}

We define a square grid $\mathbf{x}_{m_{1}, m_{2}}=\left(x_{m_{1}}, y_{m_{2}}\right)=\left(m_{1} h, m_{2} h\right)$ with step size $h$ and assume that the sides of the square $\bar{D}^{0}$ lie on the grid lines as shown in Figure 2(a). The regular grid is used to form square elements with a node at each corner. Let us define the grid set $M^{0}$ as that containing all nodes $m$ lying within the domain $D^{0}$ and also denote $N^{0}$ as the set of all nodes lying within $D^{0}$ or upon its boundary $\Gamma^{0}$.

Let us now introduce the following grid sets, $\gamma_{c}, I_{H}, I_{F}$ and $I_{F}^{\prime} \cdot \gamma_{c}$ is defined as the grid set containing all nodes lying on the crack boundary, $\Gamma_{c}$, or for which their support is intersected by the crack boundary, as shown in Figure 2(a). Now let us define a region $D^{F}$ of characteristic length $d_{F}$ such that $D^{F} \subset D^{0}$ and such that the centre point of $D^{F}$ is at the crack tip. Here we use a square domain for $D^{F}$.We define $I_{F}^{\prime}$ as the set of all nodes lying entirely within the region $D_{F}$ as seen in Figure 2(b). Next we define the grid set $I_{F}$ to include all nodes lying within $D^{F}$ along with all nodes whose support is intersected by the boundary of $D^{F}$. Therefore we have $I_{F}^{\prime} \subset I_{F}$. We also define $I_{H}$ as the set of nodes belonging to $\gamma_{c}$ but not $I_{F}^{\prime}, I_{H}=\gamma_{c} \backslash\left(I_{F}^{\prime} \bigcap \gamma_{c}\right)$. Therefore $I_{H} \subset \gamma_{c}$. Finally, we define $I_{F H}=I_{F} \bigcup I_{H}$ and the total enriched area $D_{F H}=D_{F} \bigcup D_{H}$, where $D_{H}$ is the area occupied by all elements with all four nodes belonging to $I_{H}$.

Now consider the domain $D$ in Figure 2(a), bounded by $\Gamma=\Gamma^{\prime} \cup \Gamma_{c}$. By $D^{-}$we denote the domain $D^{-}=D^{0} \backslash \bar{D}$. Considering the discretized domain, by $M^{+}$we denote the set of points $m \in M^{0}$ lying in the interior of $D$ and not on its boundary. By $N^{+}$we denote the grid set formed by the extension of $M^{+}$via the nine-node stencil (7), $N^{+}=\bigcup N_{m}, m \in M^{+}$. Similarly let us denote by $M^{-}$the set of points $m \in M^{0}$ belonging to $D^{-}$, therefore $M^{-}=M^{0} \backslash M^{+}$. By $N^{-}$we denote the extension of $M^{-}$via the stencil, $N^{-}=\bigcup N_{m}, m \in M^{-}$. It is clear that $M^{+}$and $M^{-}$do not intersect, $M^{+} \cap M^{-}=\emptyset$. We form a boundary grid set $\gamma^{\prime}$ from the intersection of the grid domains 
$N^{+}$and $N^{-}, \gamma^{\prime}=N^{+} \bigcap N^{-}$. A section of the $\gamma^{\prime}$ grid set is shown in Fig. 2(a). The entire boundary grid set is then given by $\gamma=\gamma^{\prime} \bigcup \gamma_{c}$.

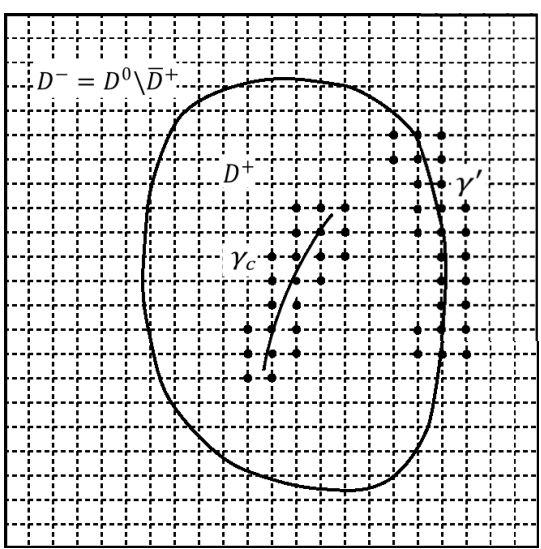

(a)

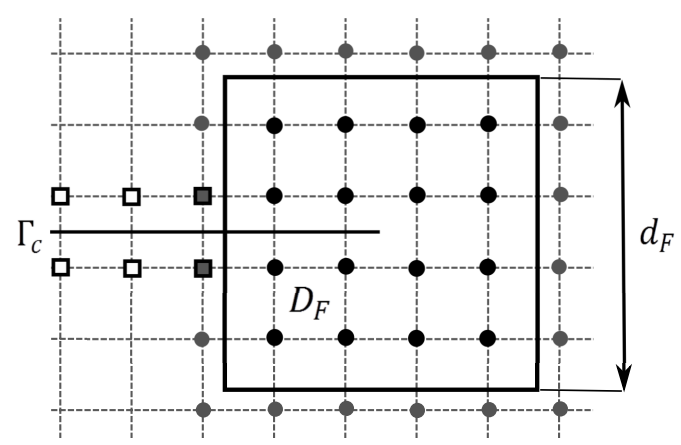

(b)

Figure 2. (a) Auxiliary domain and boundary grid sets and (b) Grid sets $I_{F}^{\prime}$ (plane nodes), $I_{F}$ (plane and gray nodes) and $I_{H}$ (square nodes)

\subsection{The Extended Finite Element Method}

In [12], an XFEM formulation for it's application with the DPM is outlined. For the test cases solved in this paper an adjusted XFEM is used. This adjusted XFEM differs from the more traditional XFEM described in [12] in that the singular enrichment functions $F^{j}$ are used along the entire length of the crack, replacing the Heaviside enrichment. This formulation allows us to consider $c_{\bar{D}}$ along the entire crack boundary. In the case that the traditional XFEM formulation with a Heaviside function is used away from the crack tip, as done in [12], a blending from the singular enrichment to the Heaviside enrichment would be required. However, it can be shown that a Heaviside enrichment can actually be replaced by a $\sqrt{r} \sin (\theta / 2)$ enrichment. In this case, orders of convergence for linear elements will be identical to those of FEM; of order 2 for the L2 norm in displacements and 1 for the H1 norm, with no loss of accuracy. For quadratic elements however, the orders of convergence will be lower than those of FEM, reduced to 2.5 for the $\mathrm{L} 2$ norm and to 1.5 for the $\mathrm{H} 1$ norm with higher error levels. This result is expected as adding a $r^{3 / 2} \sin (\theta / 2)$ term to the interpolation space is required to recover an optimal order of convergence for quadratic elements. With the choice made here on separating Mode I and Mode II displacements, for simplicity, normal opening in case of Mode I and tangent sliding in Mode II are allowed even far away from the crack, with optimal accuracy for linear elements. While introducing the Heaviside enrichment should not present a significant problem to configure, the adjusted XFEM is used here for simplicity of formulation.

The adjusted XFEM displacement used in this paper is given by:

$$
v^{(j)}=\sum_{n=1}^{\left|N^{0}\right|} a_{n}^{(j)} \psi_{n}+\sum_{n \in I_{F H}} c_{n}^{(j)} F^{(j)} \psi_{n}, \quad a_{n}^{(j)}, c_{n}^{(j)} \in \mathbb{R}, \quad j=(1,2),
$$

Here, $\psi_{n}$ are first order shape functions and $|\cdot|$ denotes the number of nodes within the grid set. $F^{(1)}$ and $F^{(2)}$ are global singular functions which are chosen by considering the problem to 
be solved. For example, for a Mode I problem solved, these functions are chosen to be the Mode I asymptotic displacement fields (3) with a SIF $K_{I}$ set to 1 . Similarly, for a Mode II test case the Mode II displacement fields (4) are used. This is a convenient formulation for simple test cases where the nature of the displacement field is well understood. For more complex test cases involving mixed mode loading, additional singular terms would need to be added to the system to account for both modes simultaneously.

This first order FEM formulation uses a nine-node stencil:

$N_{m}=\left\{\left(x_{m_{1}}, y_{m_{2}}\right),\left(x_{m_{1}} \pm h, y_{m_{2}}\right),\left(x_{m_{1}}, y_{m_{2}} \pm h\right),\left(x_{m_{1}} \pm h, y_{m_{2}} \pm h\right),\left(x_{m_{1}} \pm h, y_{m_{2}} \mp h\right)\right\}$

For more details on the original XFEM formulation, including numerical integration techniques used near the crack, see [12].

\subsection{Regular auxiliary problem with enrichment}

Consider the adjusted XFEM formulation outlined in Section 3.2. Let us introduce $V_{N^{0}}$ as the vector space of all functions $v_{N^{0}}=\left\{v_{N^{0}}^{(1)}, v_{N^{0}}^{(2)}\right\}$, defined for all $n \in N^{0}$, that satisfy the auxiliary boundary conditions $\mathrm{l}_{\Gamma^{0} N^{0}} v_{N^{0}}=0$ and are two-valued if $n \in I_{F H}$, such that:

$$
\left.v_{N^{0}}\right|_{n}= \begin{cases}0 & \text { if } n \in \Gamma^{0}, \\ a_{n} & \text { if } n \notin I_{F H} \cup \Gamma^{0}, \\ \left(c_{n}, a_{n}\right) & \text { if } n \in I_{F H} .\end{cases}
$$

Consider a case in which all enriched DOFs belonging to $\gamma_{c}$ are deactivated while any enriched DOFs outside of $\gamma_{c}$ remain active. $v_{N^{0}}$ would then be as follows:

$$
\left.v_{N^{0}}\right|_{n}= \begin{cases}0 & \text { if } n \in \Gamma^{0}, \\ a_{n} & \text { if } n \notin I_{F H} \cup \Gamma^{0}, \\ \left(0, a_{n}\right) & \text { if } n \in \gamma_{c}, \\ \left(c_{n}, a_{n}\right) & \text { if } n \in I_{F H} \backslash \gamma_{c} .\end{cases}
$$

As the enriched DOFs along the crack have been deactivated, the function $v_{N^{0}}$ in this case must be regular across the crack, i.e. no discontinuity exists. However, the singular enrichment within $I_{F}^{\prime}$ remains within the solution space. Let us define the space $U_{N^{0}}^{*} \subset V_{N^{0}}$ as the space of all functions $u_{N^{0}}^{*} \in V_{N^{0}}$ for which all enriched DOFs belonging to $\gamma_{c}$ only are deactivated, as defined by (9). Let us also define the space $U_{N^{0}}$ as that of all regular functions $u_{N^{0}}$ for which all enriched DOFs are deactivated such that $U_{N^{0}} \subset U_{N^{0}}^{*}$. Here we use the superscript $*$ to distinguish between the discrete regular space with singular enrichment, $U_{N^{0}}^{*}$ away from the crack boundary, and that without, $U_{N^{0}}$.

Let us introduce the function $\theta_{Z^{\prime}} Z$ where $Z^{\prime}$ and $Z$ are two arbitrary grid sets such that when $Z \subset Z^{\prime}$ :

$$
\left.\theta_{Z^{\prime} Z} f_{Z}\right|_{n}=\left\{\begin{array}{l}
f_{Z}, \quad \text { if } n \in Z, \\
0, \quad \text { if } n \in Z^{\prime} \backslash Z
\end{array}\right.
$$


and when $Z^{\prime} \subset Z$ :

$$
\left.\theta_{Z^{\prime} Z} f_{Z}\right|_{n}=f_{Z}, \quad \text { if } n \in Z^{\prime} .
$$

We define $F_{M^{0}}$ as the space of all body force terms $f_{M^{0}}$ for which the operator $f_{M^{0}}=\theta_{M^{0} M^{+}} f_{M^{+}}$ is defined such that:

$$
\left.\left.f_{M^{0}}\right|_{m} \equiv\left(\theta_{M^{0} M^{+}} f_{M^{+}}\right)\right|_{m}=\left\{\begin{array}{l}
f_{M^{+}}, \quad \text { if } m \in M^{+}, \\
0, \quad \text { if } m \in M^{0} \backslash M^{+} .
\end{array}\right.
$$

We now define our regular auxiliary problem with singular enrichment as:

$$
\mathbf{L}_{M^{0} N^{0}} u_{N^{0}}^{*}=f_{M^{0}}, \quad u_{N^{0}}^{*} \in U_{N^{0}}^{*}, \quad f_{M^{0}} \in F_{M^{0}},
$$

where as all functions $u_{N^{0}}^{*}$ satisfy the auxiliary boundary conditons, the problem (13) is well defined. The function $u_{N^{0}}^{*} \in U_{N^{0}}^{*}$ is therefore unique for any given body force term $f_{M^{0}}$.

\section{DIFFERENTIAL POTENTIAL AND CLEAR TRACE}

In this paper we will seek to approximate the differential potential given by:

$$
\begin{gathered}
\mathbf{P}_{\bar{D} \Gamma} \xi_{\Gamma}=v_{\bar{D}}-\mathbf{G}_{\bar{D} D} \mathbf{L}_{D \bar{D}} v_{\bar{D}}, \\
\text { or } \\
w_{\bar{D}}=v_{\bar{D}}-u_{\bar{D}} .
\end{gathered}
$$

For the origin of this expression the reader is referred to [12].

Taking the trace $\operatorname{Tr}_{\Gamma \bar{D}} w_{\bar{D}}$ of the differential potential (14) we arrive at the Boundary Equation with Projection (BEP):

$$
\mathbf{P}_{\Gamma} \xi_{\Gamma}=\xi_{\Gamma}-\operatorname{Tr}_{\Gamma \bar{D}} \mathbf{G}_{\bar{D} D} \mathbf{L}_{D \bar{D}} v_{\bar{D}}
$$

An approximation of this equation will be used to calculate the trace of the solution $v_{\bar{D}}$ on the boundary $\Gamma$.

It can be shown that if (2) is well conditioned, then (15) is also well conditioned [1].

\subsection{Clear trace for crack problems}

Consider a decomposition of the solution to the cracked auxiliary problem, $v_{\bar{D}^{0}}$, similar to that used for the adjusted XFEM (Section 3.2):

$$
v_{\bar{D}^{0}}=a_{\bar{D}^{0}}+c_{\bar{D}^{0}} F,
$$

where $a_{\bar{D}^{0}}$ and $c_{\bar{D}^{0}}$ are regular functions and $F$ is discontinuous with singular gradients of order $1 / \sqrt{r}$. Here $\operatorname{supp} c_{\bar{D}^{0}}=D_{F H}$, where $D_{F H}$ is the entire enrichmed area as defined in Section 3.2. In fact, let $F$ be equal to the global singular functions $F=\left(F^{(1)}, F^{(2)}\right)$ used within the XFEM formulation and we have $v_{\bar{D}^{0}} \in V_{\bar{D}^{0}}$. In the case where $\left.c_{\bar{D}^{0}}\right|_{\Gamma_{c}}=0$ and $\left.\frac{\partial c_{\bar{D}^{0}}}{\partial \mathbf{n}}\right|_{\Gamma_{c}}=0$, assuming that $F$ fully accounts for the discontinuity, the solution $v_{\bar{D}^{0}}$ is a regular function which is smooth and 
continuous across the crack. Therefore in this case $v_{\bar{D}^{0}} \in U_{\bar{D}^{0}}$, i.e. $v_{\bar{D}^{0}}$ is a solution to the regular auxiliary problem (5). As the solution to the regular auxiliary problem is unique, then in this case $v_{\bar{D}^{0}}=u_{\bar{D}^{0}}$ for any particular force term $f_{D^{0}}$.

Now let us consider the differential potential as defined by Ryaben'kii [1] over $D^{0}$ associated to such a function $v_{\bar{D}^{0}} \in U_{\bar{D}^{0}}$ when considering a crack boundary $\Gamma_{c}$ only. We have:

$$
\begin{aligned}
\mathbf{P}_{\bar{D}^{0} \Gamma_{c}} \xi_{\Gamma_{c}} & =v_{\bar{D}^{0}}-\mathbf{G}_{\bar{D}^{0} D^{0}} \mathbf{L}_{D^{0} \bar{D}^{0}} v_{\bar{D}^{0}}, \\
& =v_{\bar{D}^{0}}-u_{\bar{D}^{0}} \\
& =0
\end{aligned}
$$

where $\mathbf{P}_{\bar{D}^{0} \Gamma_{c}}$ is a projection matrix and $\mathbf{G}_{\bar{D}^{0} D^{0}}$ is the Green's operator, defined such that $u_{\bar{D}^{0}}=$ $\mathbf{G}_{\bar{D}^{0} D^{0}} f_{D^{0}}$.

Therefore, when the Cauchy data of the function, $c_{\bar{D}^{0}}$, is zero on $\Gamma_{c}$, i.e. $\left.c_{\bar{D}^{0}}\right|_{\Gamma_{c}}=0$ and $\left.\frac{\partial c_{\bar{D} 0}^{0}}{\partial \mathbf{n}}\right|_{\Gamma_{c}}=0$, then the differential potential due to the crack is also zero. The Cauchy data of $c_{\bar{D}^{0}}$ is therefore sufficient to supply a clear trace along the crack boundary.

Until now the crack boundary as been represented by two overlapping boundaries; $\Gamma_{c}=$ $\Gamma_{c_{l}} \cup \Gamma_{c_{r}}$. However if only the smooth, continuous function $c_{\bar{D}^{0}}$ is required at the crack boundary, then $\left.c_{\bar{D}^{0}}\right|_{\Gamma_{c_{l}}}=\left.c_{\bar{D}^{0}}\right|_{\Gamma_{c_{r}}}, \frac{\partial c_{\overline{D^{0}} 0}}{\partial \mathbf{n} \mid \Gamma_{c_{l}}}=-\left.\frac{\partial c_{\bar{D} 0}}{\partial \mathbf{n}}\right|_{\Gamma_{c_{r}}}$. Therefore we can represent the crack as one contour, $\Gamma_{c}=\Gamma_{c_{l}}$, with $\left.c_{\bar{D}^{0}}\right|_{\Gamma_{c}}=\left.c_{\bar{D}^{0}}\right|_{\Gamma_{c_{l}}}$ and $\left.\frac{\partial c_{\overline{D^{0}}}}{\partial \mathbf{n}}\right|_{\Gamma_{c}}=\left.\frac{\partial c_{\bar{D} 0} 0}{\partial \mathbf{n}}\right|_{\Gamma_{c_{l}}}$, where here the positive normal and tangential directions along $\Gamma_{c}$ are chosen to be those consistent with $\Gamma_{c_{l}}$.

We introduce the following differential trace for crack problems:

$$
\tilde{v}_{\Gamma}=\tilde{\mathbf{T}}_{\Gamma \bar{D}} v_{\bar{D}} \equiv \begin{cases}\left.\left(v_{\bar{D}}(\mathbf{x}), \frac{\partial v_{\bar{D}}(\mathbf{x})}{\partial \mathbf{n}}\right)\right|_{\Gamma^{\prime}} & \text { if } \mathbf{x} \in \Gamma^{\prime} \\ \left.\left(c_{\bar{D}}(\mathbf{x}), \frac{\partial c_{\bar{D}}(\mathbf{x})}{\partial \mathbf{n}}\right)\right|_{\Gamma_{c}} & \text { if } \mathbf{x} \in \Gamma_{c} .\end{cases}
$$

\section{APPROXIMATION OF THE SOLUTION AT THE BOUNDARY}

The Difference Potential, $w_{N^{+}}=\mathbf{P}_{N^{+} \gamma} \xi_{\gamma}=v_{N^{+}}-\mathbf{G}_{N^{+} M^{+}} \mathbf{L}_{M+N^{+}} v_{N^{+}}$, can be used to approximate the Differential Potential, $w_{\bar{D}}=\mathbf{P}_{\bar{D} \Gamma} \xi_{\Gamma}=v_{\bar{D}}-\mathbf{G}_{\bar{D} D} \mathbf{L}_{D \bar{D}} v_{\bar{D}}$. We seek the solution $v_{\bar{D}} \in V_{\bar{D}}$ to the BVP (2) and will use the adjusted FEM described in Section 3.2.

We introduce a basis along the boundary $\Gamma$ in the space of boundary densities $\Xi_{\Gamma}$. Here we define the density on the outer boundary $\Gamma^{\prime}, \xi_{\Gamma^{\prime}}$, as the Cauchy data of the function $v_{\bar{D}^{0}}$ such that $\xi_{\Gamma^{\prime}}=\left.\left(v_{\bar{D}^{0}}, \frac{\partial v_{\bar{D}^{0}}}{\partial \mathbf{n}}\right)\right|_{\Gamma^{\prime}}$. Along the crack boundary $\Gamma_{c}$, we define the density, $\xi_{\Gamma_{c}}$, as the Cauchy data of $c_{\bar{D}^{0}}$ such that $\xi_{\Gamma_{c}}=\left.\left(c_{\bar{D}^{0}}, \frac{\partial c_{\bar{D} 0}}{\partial \mathbf{n}}\right)\right|_{\Gamma_{c}}$. The density along the entire boundary is then given by:

$$
\xi_{\Gamma}= \begin{cases}\xi_{\Gamma^{\prime}} & \text { if } n \in \Gamma^{\prime}, \\ \xi_{\Gamma_{c}} & \text { if } n \in \Gamma_{c} .\end{cases}
$$

Consider the outer boundary. Let $\omega^{\prime}$ denote the set of points on $\Gamma^{\prime}$ where the basis functions exist such that we have a discrete space of vector densities denoted $\Xi_{\omega^{\prime}}$, where $\Xi_{\omega^{\prime}} \subset \Xi_{\Gamma^{\prime}}$. Since the density $\xi_{\Gamma^{\prime}}$ is a vector function with two components, $\xi_{\Gamma^{\prime}}=\left.\left(\xi_{0}, \xi_{1}\right)\right|_{\Gamma^{\prime}}$, the basis will consist of two sets of functions; $\Psi_{\omega_{0}^{\prime}}^{\prime}=\left(\psi_{0}^{1}, \psi_{0}^{2}, \ldots, \psi_{0}^{\left|\omega^{\prime}\right|}\right)$ and $\Psi_{\omega_{1}^{\prime}}^{\prime}=\left(\psi_{1}^{1}, \psi_{1}^{2}, \ldots, \psi_{1}^{\left|\omega^{\prime}\right|}\right)$ where $\left|\omega^{\prime}\right|$ is the 
total number of basis points on $\Gamma^{\prime}$. We define $\psi_{0}^{k}$ and $\psi_{1}^{k}$ as the vector functions $\psi_{0}^{k}=\left(\psi^{k}, 0\right)$ and $\psi_{1}^{k}=\left(0, \psi^{k}\right)$, where $k=1,2, . .\left|\omega^{\prime}\right|$, and then define the total system of basis functions on $\Gamma^{\prime}$ as $\Psi_{\omega^{\prime}}^{\prime}=\left(\Psi_{\omega_{0}^{\prime}}^{\prime}, \Psi_{\omega_{1}^{\prime}}^{\prime}\right)$. Similarly for the crack boundary, let $\omega_{c}$ denote the set of points on $\Gamma_{c}$ where the basis functions exist such that we have a discrete space of vector densities denoted $\Xi_{\omega_{c}} \subset \Xi_{\Gamma_{c}}$. We then have two sets of basis functions on the crack boundary; $\Phi_{\omega_{c 0}}=\left(\phi_{0}^{1}, \phi_{0}^{2}, \ldots, \phi_{0}^{\left|\omega_{c}\right|}\right)$ and $\Phi_{\omega_{c 1}}=\left(\phi_{1}^{1}, \phi_{1}^{2}, \ldots, \phi_{1}^{\left|\omega_{c}\right|}\right)$, where $\phi_{0}^{k}=\left(\phi^{k}, 0\right)$ and $\phi_{1}^{k}=\left(0, \phi^{k}\right)$. The total system on $\Gamma_{c}$ is then $\Phi_{\omega_{c}}=\left(\Phi_{\omega_{c 0}}, \Phi_{\omega_{c 1}}\right)$. We then define the total set of boundary points on $\Gamma$ as $\omega=\omega^{\prime} \bigcup \omega_{c}$, and the total set of basis functions as $\Psi_{\omega}=\left(\Psi_{\omega^{\prime}}^{\prime}, \Phi_{\omega_{c}}\right)$.

The boundary density can then be approximated in terms of the boundary basis as follows:

$$
\begin{aligned}
& \xi_{\Gamma^{\prime}}(s)=\sum_{k=1}^{\left|\omega^{\prime}\right|} \mathrm{v}_{0}^{k} \psi_{0}^{k}(s)+\sum_{k=1}^{\left|\omega^{\prime}\right|} \mathrm{v}_{1}^{k} \psi_{1}^{k}(s), \quad \text { if } s \in \Gamma^{\prime}, \\
& \xi_{\Gamma_{c}}(s)=\sum_{k=1}^{\left|\omega_{c}\right|} \mathrm{c}_{0}^{k} \phi_{0}^{k}(s)+\sum_{k=1}^{\left|\omega_{c}\right|} \mathrm{c}_{1}^{k} \phi_{1}^{k}(s), \quad \text { if } s \in \Gamma_{c},
\end{aligned}
$$

where $s$ is the arc length along $\Gamma$ from a predefined origin and $\mathrm{v}_{0}^{k}, \mathrm{v}_{1}^{k}, \mathrm{c}_{0}^{k}$ and $\mathrm{c}_{1}^{k}$ are coefficients which, with the basis functions, define the density $\xi_{\Gamma}$ along the entire boundary. Let us define $q_{0}^{k}=\mathrm{v}_{0}^{k}, q_{1}^{k}=\mathrm{v}_{1}^{k}$ for $k=1,2, . .,\left|\omega^{\prime}\right|$ and $q_{0}^{k+\left|\omega^{\prime}\right|}=c_{0}^{k}, q_{1}^{k+\left|\omega^{\prime}\right|}=c_{1}^{k}$ for $k=1,2, . .,\left|\omega_{c}\right|$. Let us also define $\psi_{0}^{k+\left|\omega^{\prime}\right|}=\phi_{0}^{k}, \psi_{1}^{k+\left|\omega^{\prime}\right|}=\phi_{1}^{k}$ for $k=1,2, . .,\left|\omega_{c}\right|$ such that the entire boundary basis can be expressed as:

$$
\xi_{\Gamma}(s)=\sum_{k=1}^{|\omega|} q_{0}^{k} \psi_{0}^{k}(s)+\sum_{k=1}^{|\omega|} q_{1}^{k} \psi_{1}^{k}(s) .
$$

When $\Gamma^{\prime}$ is a sufficiently smooth, closed contour, the components of $\xi_{\Gamma^{\prime}}$ can be considered to be smooth periodic functions of the arc length $s$. However, when the domain boundary contains corners, e.g. a square, the density $\xi_{\Gamma^{\prime}}$ consists of only piecewise smooth functions. As we seek $\xi_{\Gamma^{\prime}}$ to represent a displacement solution and its normal gradient, $\xi_{\Gamma^{\prime}}=\left.\left(v_{\bar{D}}, \frac{\partial v_{\bar{D}}}{\partial \mathbf{n}}\right)\right|_{\Gamma^{\prime}}$, we expect a continuous but non-smooth displacement, $\xi_{0}$, and a discontinuous gradient, $\xi_{1}$, across such corners. Moreover, if the outer boundary $\Gamma^{\prime}$ is intersected by the crack, i.e. in the case of an edge crack, the displacement and its normal gradient are likely to be non-smooth and possibly discontinuous at the point of intersection. In order to account for such discontinuities at boundary corners and edge cracks, the basis functions, $\Psi_{\omega^{\prime}}^{\prime}$, are divided into individual basis functions for each side of the domain. For example, consider the square domain shown in Figure 3(a). The set of points $\omega^{\prime}$ along the boundary is divided into five subsets, $\omega^{\prime}=\left(\omega_{l_{1}}^{\prime} \cup \omega_{l_{2}}^{\prime} \cup \omega_{b}^{\prime} \cup \omega_{r}^{\prime} \bigcup \omega_{t}^{\prime}\right)$. Upon each set of points we define an individual set of basis vector functions such that $\Psi_{\omega^{\prime}}=\left(\Psi_{\omega_{l_{1}}^{\prime}}, \Psi_{\omega_{l_{2}}^{\prime}}, \Psi_{\omega_{b}^{\prime}}, \Psi_{\omega_{r}^{\prime}}, \Psi_{\omega_{t}^{\prime}}\right)$. Here the left-hand side boundary $\Gamma_{l}^{\prime}$ is divided into two parts, above the crack, $\Gamma_{l_{1}}^{\prime}$, and below the crack, $\Gamma_{l_{2}}^{\prime}$.

\subsection{Local splines}

Local splines are used for the basis functions, $\Psi_{\omega}$. Crack problems contain a singularity at the crack tip which is likely to induce an error in the solution. Local splines are used instead of non-local 

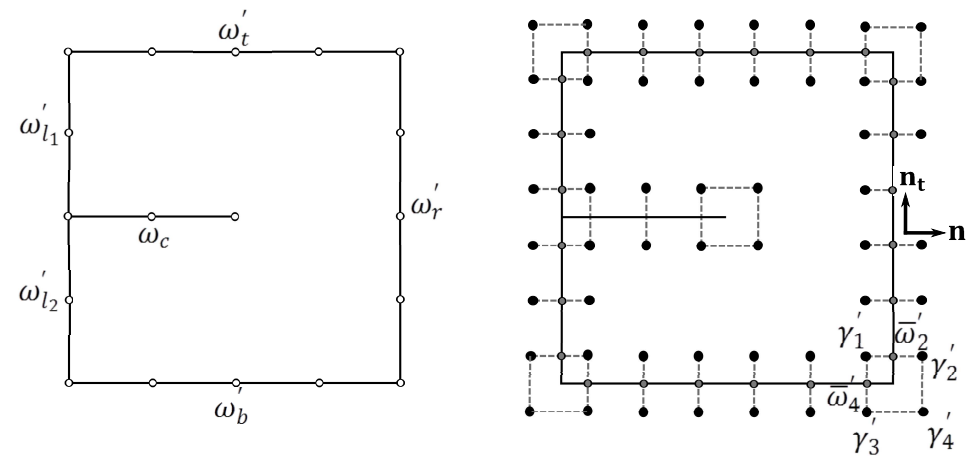

Figure 3. (a) Boundary basis points $\omega=\left(\omega_{l_{1}}^{\prime} \cup \omega_{l_{2}}^{\prime} \cup \omega_{b}^{\prime} \cup \omega_{r}^{\prime} \cup \omega_{t}^{\prime} \cup \omega_{c}\right)$, (b) Boundary points with normal through $\gamma$ node, $\bar{\omega}$, shown as gray nodes. $\gamma^{\prime}$ nodes shown in black

interpolations in an attempt to localise this error to a region near the crack tip. For simplicity of formulation these local splines are used along the entire boundary $\Gamma$.

Consider an arbitrary contour, $\Gamma_{i}$, of length $S$. Let $\omega_{i}$ be a set of equally spaced points, $s_{k}$, along the contour such that $s_{k}=(k-1) H, k=1,2, \ldots,\left|\omega_{i}\right|$, and $H=S /\left(\left|\omega_{i}\right|-1\right)$. The spline functions are piecewise polynomials which possess continuous derivatives up to the order $p$. We introduce the arbitrary function, $f_{\omega_{i}}$, which exists on $\omega_{i}$ and is equal to $f_{k}$ at the point $s_{k}$. The spline functions used in this paper to interpolate along the contour are derived from those given by Ryaben'kii in [24] and are of the form:

$$
F_{\Gamma_{i}}(s)=F_{\Gamma_{i}}\left(f_{r}, X_{k}(s), H\right), \quad r=k-s, \ldots, k+p+1-s,
$$

where $X_{k}=\left(s-s_{k}\right) / H$ and for a given $s$ the number $k$ is chosen so that the inequalities $s_{k} \leq s \leq$ $s_{k+1}$ are satisfied. This spline interpolation can be seen to be local as to calculate $F_{\Gamma_{i}}$ at any point $s$, only the values $f_{r}$ within the limits $k-s \leq r \leq k+p+q+1-s$ are used, independent of the step size $H$.

Now consider the square domain (Figure 3(a)). We have $\Gamma^{\prime}=\bigcup \Gamma_{i}^{\prime}$ for $i=\left\{l_{1}, l_{2}, b, r, t\right\}$ and $\Gamma=\Gamma^{\prime} \bigcup \Gamma_{c}$. We define the interpolation operator $\mathbf{R}_{\Gamma^{\prime} \omega^{\prime}}^{(p, H)}$ as that which extends the densities $\xi_{0}$ and $\xi_{1}$ on $\omega^{\prime}$, in addition to the tangential gradient of $\xi_{0}$, to the entire boundary $\Gamma$ using the spline interpolations (20) such that:

$$
\mathbf{R}_{\Gamma \omega}^{\prime(p, H)} \xi_{\omega}=\left.\left(\xi_{0}, \xi_{1}, \frac{\partial \xi_{0}}{\partial s}\right)\right|_{\Gamma}
$$

\subsection{Interpolation to grid nodes}

Let $\tau$ be an arbitrary grid point belonging to $\gamma^{\prime}$. Now we denote by $s_{\tau}$ the point along $\Gamma^{\prime}$ for which the normal to $\Gamma^{\prime}$ passes through the point $\tau$. We define the set of points $\bar{\omega}^{\prime}$ along the boundary as all such points $s_{\tau}$ related to each $\gamma^{\prime}$ node, as seen in Figure 3(b). Therefore each $\gamma^{\prime}$ node is associated with a point on $\Gamma^{\prime}$ belonging to $\bar{\omega}^{\prime}$. We therefore have the space of vector densities defined at $\bar{\omega}^{\prime}$; $\Xi_{\bar{\omega}^{\prime}} \subset \Xi_{\Gamma^{\prime}}$. We note that some $\gamma^{\prime}$ nodes near sharp corners, such as the node denoted as $\gamma_{1}^{\prime}$ in Figure 3(b), are related to two points on the boundary belonging to $\bar{\omega}^{\prime} ; \bar{\omega}_{2}^{\prime}$ and $\bar{\omega}_{4}^{\prime}$. In the presence of corners there may also exists $\gamma^{\prime}$ nodes for which no such point exists, for example the node $\gamma_{3}^{\prime}$ in 
Figure 3(b). We assign such nodes to the boundary points within $\bar{\omega}^{\prime}$ belonging to the neighbouring $\gamma^{\prime}$ nodes. For example, $\gamma_{3}^{\prime}$ is assigned to the boundary points $\bar{\omega}_{2}^{\prime}$ and $\bar{\omega}_{4}^{\prime}$ belonging to the $\gamma^{\prime}$ points $\gamma_{2}^{\prime}$ and $\gamma_{4}^{\prime}$ respectively. We then follow the same process on the crack boundary $\Gamma_{c}$ to form the set of points $\bar{\omega}_{c}$ associated to the grid set $\gamma_{c}$. Nodes lying beyond the crack tip are associated to the $\bar{\omega}_{c}$ points belonging to the neighbouring $\gamma_{c}$ nodes. The total set of such boundary points is defined as $\bar{\omega}=\bar{\omega}^{\prime} \bigcup \bar{\omega}_{c}$. Let $\bar{\omega}_{\tau}$ be the set of $\bar{\omega}$ points $\bar{\omega}_{\tau_{1}}, \bar{\omega}_{\tau_{2}}, \ldots$ related to each node $\tau \in \gamma$. For most $\gamma$ nodes, $\bar{\omega}_{\tau}$ consists of one boundary point only, however for nodes such as those near a corner, $\bar{\omega}_{\tau}$ can consist of multiple points.

Next we define the interpolation operator $\pi_{\gamma \bar{\omega}}^{(t)}$, where $t=0,1, \ldots, t$, in order to interpolate from the boundary points $\bar{\omega}$ to the grid nodes $\gamma$ such that:

$$
\begin{aligned}
\left.\pi_{\gamma \bar{\omega}}^{(t)} \xi_{\bar{\omega}}\right|_{\tau}=\frac{1}{\left|\bar{\omega}_{\tau}\right|} \sum_{j=1}^{\left|\bar{\omega}_{\tau}\right|}\left[\left.\xi_{\bar{D}}\right|_{\bar{\omega}_{\tau_{j}}}+\left.\rho_{n} \frac{\partial \xi_{\bar{D}}}{\partial \mathbf{n}}\right|_{\bar{\omega}_{\tau_{j}}}+\left.\rho_{t} \frac{\partial \xi_{\bar{D}}}{\partial \mathbf{n}_{t}}\right|_{\bar{\omega}_{\tau_{j}}}+\ldots\right. & \\
& \left.+\left.\sum_{k=0}^{t} \frac{\rho_{n}^{k}}{k !} \frac{\rho_{t}^{t-k}}{(t-k) !} \frac{\partial^{t} \xi_{\bar{D}}}{\partial \mathbf{n}^{k} \partial \mathbf{n}_{t}^{k-t}}\right|_{\bar{\omega}_{\tau_{j}}}\right], \\
=\frac{1}{\left|\bar{\omega}_{\tau}\right|} \sum_{j=1}^{\left|\bar{\omega}_{\tau}\right|}\left[\left.\xi_{0}\right|_{\bar{\omega}_{\tau_{j}}}+\left.\rho_{n} \xi_{1}\right|_{\bar{\omega}_{\tau_{j}}}+\left.\rho_{t} \frac{\partial \xi_{0}}{\partial \mathbf{n}_{t}}\right|_{\bar{\omega}_{\tau_{j}}}+\ldots\right. & \\
& \left.+\left.\sum_{k=0}^{t} \frac{\rho_{n}^{k}}{k !} \frac{\rho_{t}^{t-k}}{(t-k) !} \frac{\partial^{t} \xi_{\bar{D}}}{\partial \mathbf{n}^{k} \partial \mathbf{n}_{t}^{k-t}}\right|_{\bar{\omega}_{\tau_{j}}}\right], \\
t & =0,1, \ldots, \quad \tau=1, \ldots,|\gamma|,
\end{aligned}
$$

where $\rho_{n}$ and $\rho_{t}$ are the normal and tangential distances from $s_{\tau}$ to $\tau$ respectively, and the boundary normal $\mathbf{n}$ along $\Gamma$ is defined as positive when facing outwards, i.e. away from the domain, and negative when facing inwards. The positive tangential direction $\mathbf{n}_{t}$ along $\Gamma$ is defined to be the anticlockwise direction along the boundary. For the crack boundary, $\Gamma_{c}$ the positive normal and tangential directions are chosen as described in Section 4.1. Equation (22) is a Taylor expansion from the boundary to each $\tau \in \gamma$ node, averaged for each boundary point related to $\tau$.

We seek to interpolate from the boundary $\Gamma$ to find the difference trace (23) on $\gamma$ :

$$
\left.\xi_{\gamma}\right|_{n}=\left.\operatorname{Tr}_{\gamma N^{+}} v_{N^{+}}\right|_{n}= \begin{cases}a_{n} & \text { if } n \in \gamma^{\prime} \backslash \gamma_{c}^{\prime}, \\ c_{n} & \text { if } n \in \gamma_{c} \backslash \gamma_{c}^{\prime}, \\ \left(a_{n}, c_{n}\right) & \text { if } n \in \gamma_{c}^{\prime},\end{cases}
$$

The difference trace consists of the displacement components $a_{n}, n \in \gamma^{\prime}$ and $c_{n}, n \in \gamma_{c}$. However along $\Gamma^{\prime}$ we approximate the Cauchy data of $v_{\bar{D}}$ rather than $a_{\bar{D}}$. Therefore, when $n \in \gamma_{c}^{\prime}$, we interpolate to find $v_{n}$ before calculating $a_{n}$ from $a_{n}=v_{n}-F c_{n}$. Away from $\gamma_{c}$, this step is not required as $a_{n}=v_{n}$. The Cauchy data of $v_{\bar{D}}$ is considered on $\Gamma^{\prime}$ rather than that of $a_{\bar{D}}$ because high gradients tend to occur within $a_{\bar{D}}$ as one moves from the unenriched region into the enriched region, $D_{F H}$. When an edge crack is considered, these high gradients occur at the outer boundary $\Gamma^{\prime}$ and can disturb the accuracy of the interpolation of the function along the boundary, $\Gamma^{\prime} . v_{\bar{D}}$ on the other hand is smooth and continous everywhere except for across the crack boundary. This irregularity is easily accounted for by dividing $\Gamma^{\prime}$ at the intersection of $\Gamma^{\prime}$ and $\Gamma_{c}$ as described in Section 5 . We note that the density $\xi_{\omega}$ consists of two different functions, the displacement, $v_{\bar{D}^{0}}$, along $\Gamma^{\prime}$ and the 
component $c_{\bar{D}^{0}}$ along $\Gamma_{c}$. Therefore at the intersecting grid set $\gamma_{c}^{\prime}$, the grid density $\xi_{\gamma}=\pi_{\gamma \bar{\omega}}^{(t)} \xi_{\bar{\omega}}$ is two valued as shown in (23).

Now we define the operator $E x_{\gamma \omega}^{(p, H, t)}$ as that which extends the density $\xi_{\omega}$ to the $\gamma$ nodes, using the spline functions (20) to interpolate to $\bar{\omega}$ and then (22), such that $E x_{\gamma \omega}^{(p, H, t)}: \Xi_{\omega} \rightarrow \Xi_{\gamma}$, as follows:

$$
E x_{\gamma \omega}^{(p, H, t)} \xi_{\omega}= \begin{cases}E x_{\gamma^{\prime} \omega^{\prime}}^{(p, H)} \xi_{\omega^{\prime}} & =a_{n} \quad \text { if } n \in \gamma^{\prime} \backslash \gamma_{c}^{\prime}, \\ E x_{\gamma_{c} \omega_{c}}^{(p, H, t)} \xi_{\omega_{c}} & =c_{n} \quad \text { if } n \in \gamma_{c} \backslash \gamma_{c}^{\prime} \\ \left(E x_{\gamma^{\prime} \omega^{\prime}}^{(p, H, t)} \xi_{\omega^{\prime}}, E x_{\gamma_{c} \omega_{c}}^{(p, H, t)} \xi_{\omega_{c}}\right) & =\left(a_{n}, c_{n}\right) \quad \text { if } n \in \gamma_{c}^{\prime} .\end{cases}
$$

where $\Xi_{\gamma}$ is the space of all clear traces on $\gamma$.

We can then approximate the differential potential on the grid $N^{+}$as follows:

$$
\mathbf{P}_{N^{+} \gamma} E x_{\gamma \omega}^{(p, H, t)} \xi_{\omega} \approx \theta_{N^{+} \bar{D}} \mathbf{P}_{\bar{D} \Gamma} \xi_{\Gamma}, \quad \text { for } n \in N^{+}
$$

where $\xi_{\omega}=\left.\xi_{\Gamma}\right|_{\omega}$.

As can be seen in (22), a first order interpolation is readily available as the boundary densities $\xi_{0}$ and $\xi_{1}$ represent the displacement and its normal gradient on the boundary, respectively, while the tangential derivatives of these densities along the boundary are easily obtained by differentiating the basis functions:

$$
\begin{aligned}
& \frac{\partial^{t} \xi_{0}(s)}{\partial \mathbf{n}_{t}^{t}}=\frac{\partial^{t} \xi_{0}(s)}{\partial s^{t}}=\sum_{k=1}^{|\omega|} a_{0}^{k} \frac{\partial^{t} \psi_{0}^{k}(s)}{\partial s^{t}}, \\
& \frac{\partial^{t} \xi_{1}(s)}{\partial \mathbf{n}_{t}^{t}}=\frac{\partial^{t} \xi_{1}(s)}{\partial s^{t}}=\sum_{k=1}^{|\omega|} a_{1}^{k} \frac{\partial^{t} \psi_{1}^{k}(s)}{\partial s^{t}} .
\end{aligned}
$$

For higher order interpolations, an equation based interpolation can be formulated by substituting the boundary densities into the governing equation (1), as done by [15] with the Helmholtz equation.

5.2.1. Interpolation from crack boundary Along the crack boundary the function $c_{\bar{D}^{0}}$ can be interpolated without considering the singular term, $F$, without any loss of accuracy. This can be seen to be the case as follows.

Consider the function $v_{\bar{D}^{0}}^{*}=c_{\bar{D}^{0}} F$. Consider also a point $\mathbf{x}$ on the crack boundary, $\mathbf{x} \in \Gamma_{c}$, and a point $\mathbf{x}_{\tau}$ lying a distane $\rho_{n}$ from $\Gamma_{c}$ on the normal to $\Gamma_{c}$ at $\mathbf{x}$. An interpolation of $v_{\bar{D}^{0}}^{*}$ from $\mathbf{x}$ to $\mathbf{x}_{\tau}$ is given by:

$$
v_{\bar{D}^{0}}^{*}\left(\mathbf{x}_{\tau}\right)=v_{\bar{D}^{0}}^{*}(\mathbf{x})+\rho_{n} \frac{\partial v_{\bar{D}^{0}}^{*}(\mathbf{x})}{\partial \mathbf{n}}+\ldots+\sum_{k=2}^{\infty} \frac{\rho_{n}^{k}}{k !} \frac{\partial^{k} v_{\bar{D}^{0}}^{*}(\mathbf{x})}{\partial \mathbf{n}^{k}} .
$$

This Taylor expansion (27) of $v_{\bar{D}^{0}}^{*}$ can be expressed as the product of the Taylor expansion of its two components $c_{\bar{D}^{0}}$ and $F$. Therefore we have:

$$
v_{\bar{D}^{0}}^{*}\left(\mathbf{x}_{\tau}\right)=\sum_{j=0}^{\infty} \frac{\rho_{n}^{j}}{j !} \frac{\partial^{j} c_{\bar{D}^{0}}(\mathbf{x})}{\partial \mathbf{n}^{j}} \sum_{k=0}^{\infty} \frac{\rho_{n}^{k}}{k !} \frac{\partial^{k} F(\mathbf{x})}{\partial \mathbf{n}^{k}} .
$$

Let us rewrite (28) as follows: 


$$
v_{\bar{D}^{0}}^{*}\left(\mathbf{x}_{\tau}\right)=\left(\sum_{j=0}^{t} \frac{\rho_{n}^{j}}{j !} \frac{\partial^{j} c_{\bar{D}^{0}}(\mathbf{x})}{\partial \mathbf{n}^{j}}+O\left(\rho_{n}^{t+1}\right)\right) F\left(\mathbf{x}_{\tau}\right),
$$

where, as the expansion is to infinity, we have $\sum_{k=0}^{\infty} \frac{\rho_{n}^{k}}{k !} \frac{\partial^{k} F(\mathbf{x})}{\partial \mathbf{n}^{k}}=F\left(\mathbf{x}_{\tau}\right)$. As $F\left(\mathbf{x}_{\tau}\right)$ is known from the XFEM formulation, this interpolation can be used to find $c_{\bar{D}^{0}}$, and therefore $v_{\bar{D}^{0}}^{*}$, at any point $\mathbf{x}_{\tau}$ lying within the enriched area, $D_{F H}$.

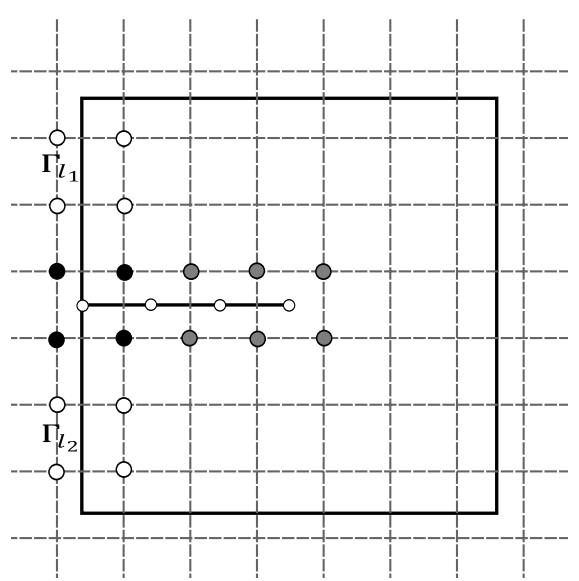

Figure 4. Edge crack problem. The grid set $\gamma_{c}^{\prime}$ shown as the plane nodes, $\gamma_{c}$ shown as gray nodes and plane nodes, and part of $\gamma^{\prime}$ shown as hollow nodes and plane nodes. Boundary basis points, $\omega_{c}$, shown along the crack boundary.

\subsection{Completion operator}

As the XFEM is a continuum method, the displacement can be found at any point within the entire domain $\bar{D}$, not only on the grid nodes $N^{0}$. The displacement at any point other than the grid nodes is found via an interpolation performed by the bilinear finite element shape functions. While this interpolation might be sufficiently accurate for the displacement function, the approximation of the displacement gradients using these shape functions will be poor. A higher order interpolation is required here between the grid nodes. We therefore introduce a completion operator which interpolates to any point within the domain $\bar{D}$ using higher order Lagrange polynomials. This completion operator will then be used to calculate the displacement and its gradients away from the grid nodes.

Let $\tau$ be a point lying anywhere within $\bar{D}$. Let us define a square $\bar{D}_{\tau}$ which contains $\tau$ and is formed by joining the four corner nodes of the nine node stencil, as defined by (7), such that $\tau \in \bar{D}_{\tau}$. The square must be defined such that the node lying at the centre of the square, $n_{c}$, belongs to $M^{+}$. Generally the choice of stencil used to form $\bar{D}_{\tau}$ is not unique. In this case we choose the stencil for which $\tau$ is closest to the centre of the square. If the choice of stencil is still not unique, we use the stencil for which $\tau$ is closest to the top left-hand corner of $\bar{D}_{\tau}$. We denote the nodes of the stencil used to form $D_{\tau}$ as $N_{\tau}$ where $N_{\tau} \in N^{+}$.

Consider an arbitrary function $z_{N_{\tau}}$ defined on $N_{\tau}$ and set the notation $z_{i, j}=z_{N_{\tau}}\left(\mathbf{x}_{i, j}\right)$. Now we define the following Lagrange polynomial at $\mathbf{x} \in \bar{D}_{\tau}$ : 


$$
\mathcal{P}_{\bar{D}_{\tau}}\left(\mathbf{x}, N_{\tau}, z_{N_{\tau}}\right)=\sum_{i=1}^{N} \sum_{j=1}^{J} l_{i, j}(\mathbf{x}) z_{i, j},
$$

where $N$ and $J$ are the total number of nodes in the $\mathrm{x}$ and y directions respectively, and the basis polynomials $l_{i, j}$ are given by:

$$
l_{i, j}(\mathbf{x})=\prod_{\substack{1 \leq m \leq N \\ m \neq i}} \frac{\eta-\eta_{m}}{\eta_{i}-\eta_{m}} \prod_{\substack{1 \leq n \leq J \\ n \neq j}} \frac{\zeta-\zeta_{n}}{\zeta_{j}-\zeta_{n}},
$$

and here $\eta=\left(x-x_{c}\right) / h$ and $\zeta=\left(y-y_{c}\right) / h$ where $\left(x_{c}, y_{c}\right)$ are the coordinates of the central node $n_{c}$. Using the nine node grid set $N_{\tau}$, such that $N=J=3$, these polynomials are second order. A larger grid set allows for higher order polynomials to be used, however second order accuracy is sufficient for the problems within this paper.

Consider a function $z_{\bar{D}}$ defined in $\bar{D}$ and set $z_{N^{+}}=\left.z_{\bar{D}}\right|_{N^{+}}$. We can approximate this function at any point $\mathbf{x}$ within $\bar{D}$ using the Lagrange polynomial (29) such that:

$$
\tilde{z}_{\bar{D}}=\mathcal{P}_{\bar{D}}\left(\mathbf{x}, N^{+}, z_{N^{+}}\right) \approx z_{\bar{D}}(\mathbf{x}) .
$$

Let us define the completion operator $\mathbb{P}_{\bar{D} N^{+}}: V_{N^{+}} \rightarrow V_{\bar{D}}$ such that using the polynomial interpolation defined by (30):

$$
\mathbb{P}_{\bar{D} N^{+}} v_{N^{+}}= \begin{cases}\tilde{a}_{\bar{D}} & \text { if } \mathbf{x} \notin D_{F H}, \\ \left(\tilde{a}_{\bar{D}}, \tilde{c}_{\bar{D}}\right) & \text { if } \mathbf{x} \in D_{F H},\end{cases}
$$

where $\tilde{a}_{\bar{D}}=\tilde{v}_{\bar{D}}=\mathcal{P}_{\bar{D}}\left(\mathbf{x}, N^{+}, v_{N^{+}}\right)$when $\mathbf{x} \notin D_{F H}$ and when $\mathbf{x} \in D_{F H}$ we have $\tilde{a}_{\bar{D}}=\tilde{v}_{\bar{D}}-$ $F \tilde{c}_{\bar{D}}$, where $\tilde{v}_{\bar{D}}=\mathcal{P}_{\bar{D}}\left(\mathbf{x}, N^{+}, v_{N^{+}}\right)$, and $\tilde{c}_{\bar{D}}=\mathcal{P}_{\bar{D}}\left(\mathbf{x}, N^{+}, c_{N^{+}}\right)$. The function $a_{\bar{D}}$ is approximated indirectly within $D_{F H}$ due to the high gradients of the function which occur at the boundary of the enriched region as discussed in Section 5.2.

5.3.1. Interpolation near crack boundary The support of the function $c_{N^{+}}$is limited to grid sets local to the crack, therefore the nine node stencil $N_{\tau}$ used for this interpolation in Section 5.3 can be too large. Therefore near the crack $\bar{D}_{\tau}$ is chosen to be the largest quadrilateral possible up to a maximum length of two elements in any direction. An example of the grid sets chosen for nodes belonging to $I_{H}$ are shown in Figure 5. Considering the second term on the right hand side of the XFEM displacement given by equation (6), we can see that $c_{\bar{D}^{0}}$ is continuous across the crack as $c_{\bar{D}^{0}}=\sum_{n \in I_{F H}} c_{n} \psi_{n}$, where $\psi_{n}$ is continuous across the element. Therefore the Lagrange interpolation across the crack is not a problem here for $c_{\bar{D}^{0}}$. However, when interpolating $v_{N^{+}}$near the crack boundary, $N_{\tau}$ is chosen such that $\bar{D}_{\tau}$ is not intersected by the crack as $v_{N^{+}}$is discontinuous across the crack.

\subsection{Approximation of Differential Potential and Boundary Projection}

We can now therefore approximate the differential potential (14) at any point $\mathrm{x} \in \bar{D}$ as follows: 


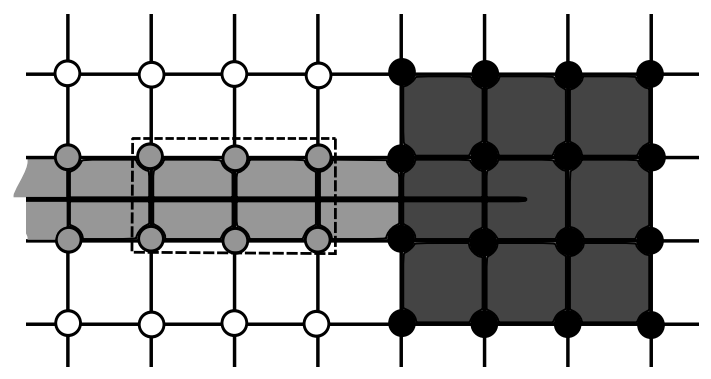

Figure 5. Stencil used for Lagrange polynomial near crack boundary. Nodes belonging to $I_{H}$ shown in grey, nodes belonging to $I_{F}$ shown in black.

$$
\mathbb{P}_{\bar{D} N^{+}} \mathbf{P}_{N^{+} \gamma} E x_{\gamma \omega}^{(p, H, t)} \xi_{\omega} \approx \mathbf{P}_{\bar{D} \Gamma} \xi_{\gamma}
$$

In addition to interpolating a function, the polynomials (29) can be used to approximate the first derivatives of a function as follows:

$$
\frac{\partial \mathcal{P}_{\bar{D}}\left(\mathbf{x}, N^{+}, z_{N^{+}}\right)}{\partial \mathbf{x}}=\sum_{n=1}^{N} \sum_{j=1}^{J} \frac{\partial l_{i, j}(\mathbf{x})}{\partial \mathbf{x}} z_{i, j} \approx \frac{\partial z_{\bar{D}}(\mathbf{x})}{\partial \mathbf{x}} .
$$

Let us approximate the trace operator (16), $\tilde{\operatorname{Tr}}_{\Gamma N^{+}}: V_{N^{+}} \rightarrow \Xi_{\Gamma}$, using these Lagrange polynomials such that:

$$
\tilde{\mathbf{T r}}_{\Gamma N^{+}} v_{N^{+}}= \begin{cases}\left(\mathcal{P}_{\bar{D}}\left(\mathbf{x}, N^{+}, v_{N^{+}}\right), \frac{\partial \mathcal{P}_{\bar{D}}\left(\mathbf{x}, N^{+}, v_{N^{+}}\right)}{\partial \mathbf{n}}\right) & \text { for } \mathbf{x} \in \Gamma^{\prime} \\ \left(\mathcal{P}_{\bar{D}}\left(\mathbf{x}, N^{+}, c_{N^{+}}\right), \frac{\partial \mathcal{P}_{\bar{D}}\left(\mathbf{x}, N^{+}, c_{N^{+}}\right)}{\partial \mathbf{n}}\right) & \text { for } \mathbf{x} \in \Gamma_{c} .\end{cases}
$$

We can therefore approximate the BEP (15) as follows:

$$
\tilde{\mathbf{T}} \mathbf{r}_{\Gamma N^{+}} \mathbf{P}_{N^{+} \gamma} E x_{\gamma \omega}^{(p, H, t)} \xi_{\omega}=\mathbf{R}_{\Gamma \omega}^{(p, H)} \xi_{\omega}-\tilde{\mathbf{T}}_{\Gamma N^{+}} \mathbf{G}_{N^{+} M^{+}} \mathbf{L}_{M^{+} N^{+}} v_{N^{+}},
$$

where the operator $\mathbf{R}_{\Gamma \omega}^{(p, H)}$ differs slightly to $\mathbf{R}_{\Gamma \omega}^{\prime(p, H)}$ (21) in that it extends the Cauchy data only, without the tangential gradient, such that $\mathbf{R}_{\Gamma \omega}^{(p, H)}: \Xi_{\omega} \rightarrow \Xi_{\Gamma}$.

Let us also introduce an alternative BEP which has the slight difference that here the tangential gradient is calculated on the boundary $\Gamma$ in addition to the function and its normal gradient:

$$
\tilde{\mathbf{T}}_{\Gamma N^{+}}^{\prime} \mathbf{P}_{N^{+} \gamma} E x_{\gamma \omega}^{(p, H, t)} \xi_{\omega}=\mathbf{R}_{\Gamma \omega}^{\prime(p, H)} \xi_{\omega}-\tilde{\mathbf{T}}_{\Gamma N^{+}}^{\prime} \mathbf{G}_{N^{+} M^{+}} \mathbf{L}_{M^{+} N^{+}} v_{N^{+}}
$$

where the operator $\tilde{\mathbf{T r}}_{\Gamma N^{+}}^{\prime}$ is defined such that:

$$
\tilde{\mathbf{T}}_{\Gamma^{+}}^{\prime} v_{N^{+}}= \begin{cases}\left(\mathcal{P}_{\bar{D}}\left(\mathbf{x}, N^{+}, v_{N^{+}}\right), \frac{\partial \mathcal{P}_{\bar{D}}\left(\mathbf{x}, N^{+}, v_{N}\right)}{\partial \mathbf{n}}, \frac{\partial \mathcal{P}_{\bar{D}}\left(\mathbf{x}, N^{+}, v_{N^{+}}\right)}{\partial \mathbf{n}_{t}}\right) & \text { for } \mathbf{x} \in \Gamma^{\prime}, \\ \left(\mathcal{P}_{\bar{D}}\left(\mathbf{x}, N^{+}, c_{N^{+}}\right), \frac{\partial \mathcal{P}_{\bar{D}}\left(\mathbf{x}, N^{+}, c_{N^{+}}\right)}{\partial \mathbf{n}}, \frac{\partial \mathcal{P}_{\bar{D}}\left(\mathbf{x}, N^{+}, c_{N^{+}}\right)}{\partial \mathbf{n}_{t}}\right) & \text { for } \mathbf{x} \in \Gamma_{c} .\end{cases}
$$

The additional constraint of evaluating the tangential gradient of the function at the boundary serves only to apply a stricter requirement on the clear trace $\xi_{\Gamma}$ needed to satisfy the BEP. Finally, 
let us define the operator $\tilde{\mathbf{T r}}_{\bar{\omega} N^{+}}^{\prime} v_{N^{+}}$as that which provides the trace (37) on the boundary subset $\bar{\omega} \subset \Gamma$ only.

\subsection{Approximation of boundary operator}

The spline functions (20) to approximate the boundary conditions, $1_{\Gamma \bar{D}} v_{\bar{D}}=\varphi_{\Gamma}$, which we will apply in the form:

$$
\mathbf{l}_{\bar{\omega} \omega} \xi_{\omega}=\varphi_{\bar{\omega}} .
$$

The operator $\mathbf{l}_{\bar{\omega} \omega}$ can define Dirichlet, Neuman or Robin boundary conditions using these spline interpolations. These splines can be used to interpolate the function $\xi_{0}$ and its normal derivative $\xi_{1}$ along the entire boundary and can also calculate the tangential derivative $\frac{\partial \xi_{0}}{\partial \mathbf{n}_{t}}$, as described in Section 5.1.

Therefore the method holds the advantage that the boundary conditions need never be approximated on a grid. The accuracy of the boundary conditions relies only on the order of the boundary basis functions chosen, in this case the order $p=2$ of the local splines (20).

\section{NUMERICAL ALGORITHM}

Let us now formulate the solution method for general crack problems where $v_{\bar{D}} \in V_{\bar{D}}$. We seek a boundary density $\xi_{\Gamma}$ which satisfies the BEP (15). An approximate solution is obtained by finding a solution to the approximated BEP (36) along with the boundary equation (38).

Let us rewrite the approximated BEP (36) as the following set of equations evaluated on the boundary set $\bar{\omega}$ :

$$
\begin{aligned}
\sum_{k=1}^{|\omega|} q_{0}^{k} \tilde{\mathbf{T}}_{\bar{\omega} N^{+}}^{\prime} \mathbf{P}_{N^{+} \gamma} E x_{\gamma \omega}^{(p, H, t)} \psi_{0}^{k}+\sum_{k=1}^{|\omega|} q_{1}^{k} \tilde{\mathbf{T}}_{\bar{\omega} N^{+}}^{\prime} \mathbf{P}_{N^{+} \gamma} E x_{\gamma \omega}^{(p, H, t)} \psi_{1}^{k} \\
=\sum_{k=1}^{|\omega|} q_{0}^{k} \mathbf{R}_{\omega^{\prime} \omega}^{(p, H)} \psi_{0}^{k}+\sum_{k=1}^{|\omega|} q_{1}^{k} \mathbf{R}_{\bar{\omega} \omega}^{\prime(p, H)} \psi_{1}^{k}-\tilde{\mathbf{T}}_{\bar{\omega} N^{+}}^{\prime} \mathbf{G}_{N^{+} M^{+}} \mathbf{L}_{M^{+} N^{+}} v_{N^{+}}
\end{aligned}
$$

We then form the vector of unknown coefficients of length $4|\omega|$;

$$
\mathbf{q}_{\omega}=\left[\mathrm{v}_{\omega^{\prime}}, \mathbf{c}_{\omega_{c}}\right]=\left[\mathrm{v}_{0}^{1}, \ldots, \mathrm{v}_{0}^{\left|\omega^{\prime}\right|}, \mathrm{v}_{1}^{1}, \ldots, \mathrm{v}_{1}^{\left|\omega^{\prime}\right|}, c_{0}^{1}, \ldots, c_{0}^{\left|\omega_{c}\right|}, c_{1}^{1}, \ldots, c_{1}^{\left|\omega_{c}\right|}\right]
$$

We note that here each term $\mathrm{v}_{0}^{k}, \mathrm{v}_{1}^{k}, c_{0}^{k}$ and $c_{1}^{k}$ are in fact vectors containing each directional component; $\mathrm{v}_{0}^{k}=\left(\mathrm{v}_{0}^{(1) k}, \mathrm{v}_{0}^{(2) k}\right), \mathrm{v}_{1}^{k}=\left(\mathrm{v}_{1}^{(1) k}, \mathrm{v}_{1}^{(2) k}\right), c_{0}^{k}=\left(c_{0}^{(1) k}, c_{0}^{(2) k}\right)$ and $c_{1}^{k}=\left(c_{1}^{(1) k}, c_{1}^{(2) k}\right)$ such that $\mathrm{v}_{\omega^{\prime}}$ and $\mathbf{c}_{\omega_{c}}$ can be written as:

$$
\mathbf{v}_{\omega^{\prime}}=\left[\mathbf{v}_{0}^{(1) 1}, \mathbf{v}_{0}^{(2) 1}, \ldots, \mathbf{v}_{0}^{(1)\left|\omega^{\prime}\right|}, \mathbf{v}_{0}^{(2)\left|\omega^{\prime}\right|}, \mathbf{v}_{1}^{(1) 1}, \mathbf{v}_{1}^{(2) 1}, \ldots, \mathbf{v}_{1}^{(1)\left|\omega^{\prime}\right|}, \mathbf{v}_{1}^{(2)\left|\omega^{\prime}\right|}\right]
$$

and 


$$
\mathbf{c}_{\omega_{c}}=\left[c_{0}^{(1) 1}, c_{0}^{(2) 1}, \ldots, c_{0}^{(1)\left|\omega_{c}\right|}, c_{0}^{(2)\left|\omega_{c}\right|}, c_{1}^{(1) 1}, c_{1}^{(2) 1}, \ldots, c_{1}^{(1)\left|\omega_{c}\right|}, c_{1}^{(2)\left|\omega_{c}\right|}\right]
$$

respectively.

We then represent the system of equations (39) in matrix form:

$$
\mathbf{D}_{\bar{\omega} \omega} \mathbf{q}_{\omega}=-\tilde{\mathbf{T}}_{\bar{\omega} N^{+}}^{\prime} \mathbf{G}_{N^{+} M^{+}} \mathbf{L}_{M^{+} N^{+}} v_{N^{+}} .
$$

where the matrix operator $\mathbf{D}_{\bar{\omega} \omega}$ of dimensions $6|\bar{\omega}| \times 4|\omega|$ is as follows:

$$
\mathbf{D}_{\bar{\omega} \omega}=\left[\tilde{\mathbf{D}}_{\bar{\omega} \omega} \psi_{0}^{1}, \ldots, \tilde{\mathbf{D}}_{\bar{\omega} \omega} \psi_{0}^{|\omega|}, \tilde{\mathbf{D}}_{\bar{\omega} \omega} \psi_{1}^{1}, \ldots, \tilde{\mathbf{D}}_{\bar{\omega} \omega} \psi_{1}^{|\omega|}\right]
$$

with $\tilde{\mathbf{D}}_{\bar{\omega} \omega}=\tilde{\mathbf{T}}_{\bar{\omega} N^{+}}^{\prime} \mathbf{P}_{N^{+} \gamma} E x_{\gamma \omega}^{(p, H, t)}-\mathbf{R}_{\bar{\omega} \omega}^{\prime(p, H)}$.

Each column of $\mathbf{D}_{\bar{\omega} \omega}$ is found by first extending each individual basis function on $\omega$ to the $\gamma$ grid set before calculating the difference potential (25). Each time the difference potential is calculated a solution of the auxiliary problem (13) is required. Therefore in order to form $\mathbf{D}_{\bar{\omega} \omega}, 4|\omega|$ solutions of the auxiliary problem is needed. One additional solution of the auxiliary problem is also needed to calculate the right-hand side of (41). However for homogeneous problems, $\mathbf{L}_{\bar{D} D} v_{\bar{D}}=0_{D}$, this solution is not needed. The difference potential is then reduced to the boundary points $\bar{\omega}$ using the Lagrange interpolations (29) where the function and its first derivatives are evaluated.

We therefore have a system of linear algebraic equations (41). Generally it is found that $|\omega|<|\bar{\omega}|$ for meaningful solutions. Therefore we have an overdetermined system which can be solved using least squares. Solving the system (41) along with the boundary conditions (38) leads to an unique solution.

The difference potential, $w_{N^{+}}$, can then be found from (25) using the calculated boundary density $\xi_{\omega}$ before the final solution is obtained from:

$$
v_{N^{+}}=w_{N^{+}}+u_{N^{+}}
$$

where $u_{N^{+}}=\mathbf{G}_{N^{+} M^{+}} \mathbf{L}_{M^{+} N^{+}} v_{N^{+}}=\mathbf{G}_{N^{+} M^{+}} f_{M^{+}}$.

\subsection{Summary of computational procedure}

A step-by-step summary of the computational procedure is as follows:

1. Define the discrete auxiliary problem (13). The auxiliary problem must be well posed with a domain that fully contains the embedded domain, $\bar{D}$, and its boundary, $\Gamma$. The auxiliary boundary conditons $\mathrm{l}_{\Gamma^{0} N^{0}} u_{N^{0}}^{*}=0$ can be chosen as to facilitate the most efficient solution method.

2. Define the required grid sets $\gamma, \gamma^{\prime}, \gamma_{c}, \gamma_{c}^{\prime}, I_{F H}, N^{+}, M^{+}$.

3. Define a suitable system of basis functions along the boundary in order to approximate the boundary density $\xi_{\Gamma}$ as in (19). Each basis function, $\psi_{0}^{k}, \psi_{1}^{k}$, can be defined using any properties known for the solution a priori. In this paper all basis functions are defined using the local spline functions given in Section 5.1. 
4. Build the boundary system of equations (41). For each directional component $j=(1,2)$, we do the following for $k=1,2, \ldots,|\omega|$ and $i=0,1$ :

4.1. Extend the boundary basis function $\psi_{i}^{k}$ to the grid boundary $\gamma$ using the extension operator (24) to find the grid density $\xi_{\gamma}^{k, i}$.

4.2. Calculate the difference potential $w_{N^{+}}^{k, i}=v_{N^{+}}^{k, j}-\mathbf{G}_{N^{+} M^{+}} \mathbf{L}_{M^{+} N^{+}} v_{N^{+}}^{k, j}$ for $v_{N^{+}}^{k, j}=$ $\theta_{N^{+}+} \xi_{\gamma}^{k, i}$. This requires a solution of the auxiliary problem (13) with right-hand side $f_{M^{0}}^{k, i}=\mathbf{L}_{M^{0} N^{0}} \theta_{N^{0} N^{+}} v_{N^{+}}^{k, i}$.

4.3. Calculate the trace (37) of $w_{N^{+}}^{k, i}$ on the boundary points $\bar{\omega}$ using the Lagrange interpolations and store as a column of matrix $\mathbf{D}_{\bar{\omega} \omega}$.

5. Solve the auxiliary problem (13) with right-hand side $\theta_{M^{0} M^{+}} f_{M^{+}}$to find $u_{N^{+}}$. If the problem is homogeneous such that $\mathbf{L}_{M^{+} N^{+}} v_{N^{+}}=f_{M^{+}}=0_{M^{+}}$, then this step is not required.

6. Solve the boundary system (41) with the boundary conditions $\mathbf{l}_{\bar{\omega} \omega} \xi_{\omega}=\varphi_{\bar{\omega}}$, where $\mathbf{l}_{\bar{\omega} \omega}$ can be approximated as described in Section 5.5, to find the boundary density $\xi_{\omega}$.

7. Extend the boundary density, $\xi_{\omega}$, to $\gamma$ to find the grid density, $\xi_{\gamma}$. Calculate the difference potential $w_{N^{+}}=v_{N^{+}}-\mathbf{G}_{N^{+} M^{+}} \mathbf{L}_{M^{+} N^{+}} v_{N^{+}}$where $v_{N^{+}}=\theta_{N^{+} \gamma} \xi_{\gamma}$. This requires the solution of the auxiliary problem (13) with right-hand side $\mathbf{L}_{M^{0} N^{0}} \theta_{N^{0} N^{+}} v_{N^{+}}$.

8. The final solution is then found from $v_{N^{+}}=w_{N^{+}}+u_{N^{+}}$.

The majority of the computational effort needed within the procedure exists within Step 4 where $4|\omega|$ solutions of the auxiliary problem are required. When solving multiple problems on the same domain, $\bar{D}$, but with different boundary conditions or force terms, the BEP can be stored and reused for each problem. In this case, only two solutions of the auxiliary problem is needed, Steps 5 and 7 , to find any additional solution once the BEP is known. Therefore, the cost of Step 4 becomes less essential when multiple problems are to be solved.

The cost involved in constructing the BEP is not as significant as might first appear. As is shown in Section 7, the number of basis functions, $|\omega|$, doesn't necessarily need to increase with increasing mesh refinement. Therefore, particularly for smooth boundary densities, it is expected that $|\omega|<<\left|N^{0}\right|$. We have shown in this paper (Section 4.1) that only smooth, regular functions are required even along the crack boundary. Further to this, a major advantage of the DPM is its ability to solve any problem using a regular grid, regardless of the complexity of the problem's geometry. This is a key advantage over classical FEM for two reasons; the first is the avoidance of generating a mesh which conforms with the domain boundaries, and the second is avoiding the expensive Gauss quadrature procedure for the construction of the stiffness matrix. The Gauss quadrature can be avoided as the regular grid allows the stiffness matrix to be defined explicitly, e.g. using a finite difference formulation. Therefore the cost of the $4|\omega|+2$ required solutions of the auxiliary problem is compensated by the avoidance of these expensive steps. In Section 8 an alternative solution algorithm for the auxiliary problem is provided. The algorithm has the potential to be fast, particularly for problems with small enrichment areas, reducing the cost of the DPM.

The solution of the auxiliary problem in Step 7 can be avoided if in Step 4 we also construct the projection matrix $\mathbf{P}_{N+\omega}=\mathbf{P}_{N^{+} \gamma} E x_{\gamma \omega}^{(p, H, t)}$. The projection matrix can be constructed by storing each difference potential, $w_{N^{+}}^{k, i}$, in Step 4.2. as a column of the matrix $\mathbf{P}_{N^{+} \omega}$ such that: 


$$
\begin{aligned}
\mathbf{P}_{N^{+} \omega}=\left[\mathbf{P}_{N^{+} \gamma} E x_{\gamma \omega}^{(p, H, t)} \psi_{0}^{1}, \ldots, \mathbf{P}_{N^{+} \gamma} E x_{\gamma \omega}^{(p, H, t)} \psi_{0}^{|\omega|},\right. & \\
& \left.\mathbf{P}_{N^{+} \gamma} E x_{\gamma \omega}^{(p, H, t)} \psi_{1}^{1}, \ldots, \mathbf{P}_{N^{+} \gamma} E x_{\gamma \omega}^{(p, H, t)} \psi_{1}^{|\omega|}\right] .
\end{aligned}
$$

The difference potential can then be obtained from (25), $w_{N^{+}}=\mathbf{P}_{N^{+}} \xi_{\omega}$, without requiring an additional solution of the auxiliary problem.

\section{CONVERGENCE TESTS}

\subsection{Test cases}

Before solving a crack test case, we solve a regular problem in order to demonstrate the optimal accuracy of the solution method when no singularity or discontinuity exists within the problem. The regular problem to be solved is that of a $2 \mathrm{D}$, in plane bending of a beam. The analytical solution for this problem is known and is given by:

$$
\begin{gathered}
u^{(1)}=x y, \\
u^{(2)}=-\left(x^{2}+\nu y^{2}\right) / 2 .
\end{gathered}
$$

Dirichlet boundary conditions are applied upon the entire boundary such that $\left.\xi_{0}^{(1)}\right|_{\Gamma}=\left.u^{(1)}\right|_{\Gamma}$ and $\left.\xi_{0}^{(2)}\right|_{\Gamma}=\left.u^{(2)}\right|_{\Gamma}$. The domain is square and therefore must deal with a boundary containing sharp corners.

Two crack problems are then solved, the Mode I (3) and Mode II (4) asymptotic displacement fields over a square domain with an edge crack, as in Figure 4. These problems contain singular derivatives at the crack tip and a discontinuity across the crack boundary. Dirichlet boundary conditions are applied along the outer boundary, $\Gamma^{\prime}$, such that $\left.\xi_{0}^{(1)}\right|_{\Gamma^{\prime}}=\left.v_{j}^{(1)}\right|_{\Gamma}$ and $\left.\xi_{0}^{(2)}\right|_{\Gamma^{\prime}}=\left.v_{j}^{(2)}\right|_{\Gamma}$ for $j=I$ for the Mode I problem and $j=I I$ for the Mode II problem. Along the crack, free traction boundary conditions are applied such that $\left.\mathbf{t}\left(\xi_{0}, \xi_{1}\right)\right|_{\Gamma_{c}}=0_{\Gamma_{c}}$.

\subsection{Accuracy}

The relative $L^{2}$ norm, $\left(\left\|v^{h}-v\right\|_{L^{2}}\right) /\left(\|v\|_{L^{2}}\right)$, and relative Sobolev norm $\left(H^{1}\right)$, $\left(\| v^{h}-\right.$ $\left.v \|_{H^{1}}\right) /\left(\|v\|_{H^{1}}\right)$, as defined in [12] are approximated for the convergence study where $v^{h}$ is the approximated solution and $v$ is the known analytical solution.

Ryaben'kii [1] shows that the accuracy for a difference potential approximating a second order differential operator, $\mathbf{L}_{D^{0}} \bar{D}^{0}$, is $O\left(h^{\mathrm{p}}\right)$, where $\mathrm{p}$ is the accuracy of the difference operator $\mathbf{L}_{M^{0}} N^{0}$. Therefore we expect:

$$
\left\|\theta_{N^{+} \bar{D}^{0}} v_{\bar{D}^{0}}-P_{N^{+}} \xi_{\gamma}\right\|_{L^{2}+\varepsilon} \leq \mathrm{const} \cdot h^{2-\varepsilon},
$$

where $\varepsilon$ is arbitrary, with $0<\varepsilon<1$, and represents the error in approximating the norm.

However the convergence of the approximated differential potential (32) depends not only on the accuracy of the auxiliary problem, but also on each interpolation used to extend the density from 
the boundary to the grid nodes and then back to the boundary. If the boundary extension operator, $E x_{\gamma \omega}^{(p, H, t)}$, in (36) in addition to the trace operator, $\tilde{\mathbf{T r}}_{\Gamma N^{+}}^{\prime}$, provides second order approximations or higher then one would expect to achieve an optimal convergence rate of 2 for the $L^{2}$ norm. Therefore for the solution of these test cases we use $p=2, t=2$ such that we have $E x_{\gamma \omega}^{(2, H, 2)}$. We also use second order Lagrange polynomials for the trace operator, $\tilde{\operatorname{Tr}}_{\Gamma N^{+}}^{\prime}$, where possible. At the crack boundary, first order polynomials are used where a restricted number of enriched nodes are available, as discussed in Section 5.3.1. However, as the crack test cases to be solved consist of a horizontal, straight line crack, we can achieve a second order approximation by ensuring that the crack lies exactly halfway through the elements. In this way, the first order polynomials provide a second order approximation.

These crack test cases were also solved in [12] using a DPM formulation solved at the $\gamma$ nodes. Here it was observed that with geometrical enrichment, i.e. a constant enrichment area $D_{F}$, nearoptimal convergence rates of near 2 were achieved for both the $L^{2}$ and $H^{1}$ norms. The high convergence rate of 1.8 achieved for the $H^{1}$ norm is due to symmetry properties of the problem solved and the regular grid used, as observed by $[25,26]$. This superconvergence is observed for the regular problem solved in this paper.

\subsection{Convergence results}

7.3.1. Regular test case Before performing a convergence study for the grid refinement of the auxiliary problem, it is necessary to determine the number of boundary points $|\omega|$ needed to ensure that the convergence rate is independent of $|\omega|$. In this way we obtain an accurate insight into the performance of the method when the number of solutions to the auxiliary problem is constant despite increasing grid refinement. In order to do so, we performed a convergence study for varying $|\omega|$ using our maximum grid size $\left|N^{0}\right|=185^{2}$. It was found that for $|\omega|>240$ the accuracy of the solution for this maximum grid size became independent of $|\omega|$, as can be seen from Figure 6 .

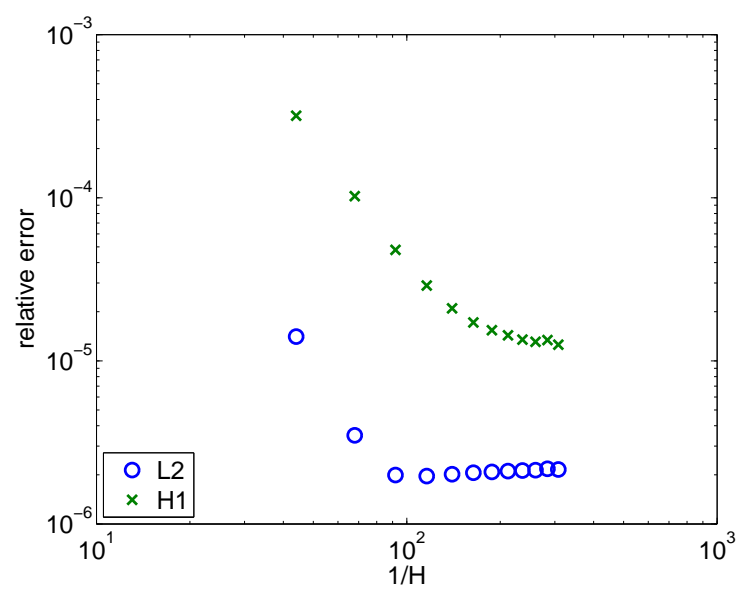

Figure 6. Error in relative $L^{2}$ and $H^{1}$ norms for the regular problem for increasing $|\omega|$ and fixed grid size of

$$
\left|N^{0}\right|=185^{2}
$$

Therefore we now perform a convergence study for grid refinement where we keep at a constant $|\omega|=260$. From Figure 7 we see that optimal convergence rates of 2 is achieved for the $L^{2}$ norm while superconvergence of 1.8 is achieved for the $H^{1}$ norm. 


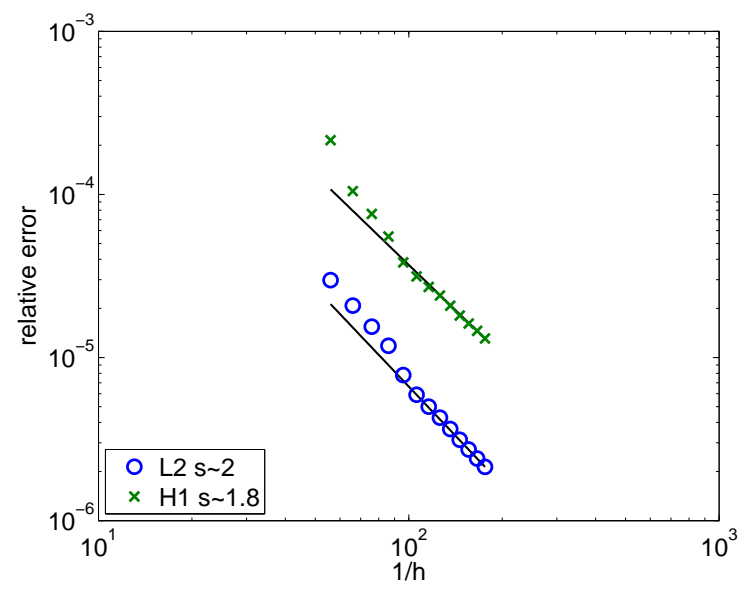

Figure 7. Error in relative $L^{2}$ and $H^{1}$ norms for the regular problem

7.3.2. Crack test cases For the crack problem the number of $\omega$ points were chosen such that the step size along the boundary, $H$, was equal to that of the regular problem. We therefore perform a convergence study for the grid refinement using $|\omega|=298$. A geometrical enrichment scheme is used. We see from Figure 8 that a near optimal convergence rates of 1.9 and 0.9 are achieved for the $L^{2}$ and $H^{1}$ norms respectively. However we see that the superconvergence of the $H^{1}$ norm seen for the regular problem is lost in this case. These results are very similar to the those obtained by Larborde et al. in [22].

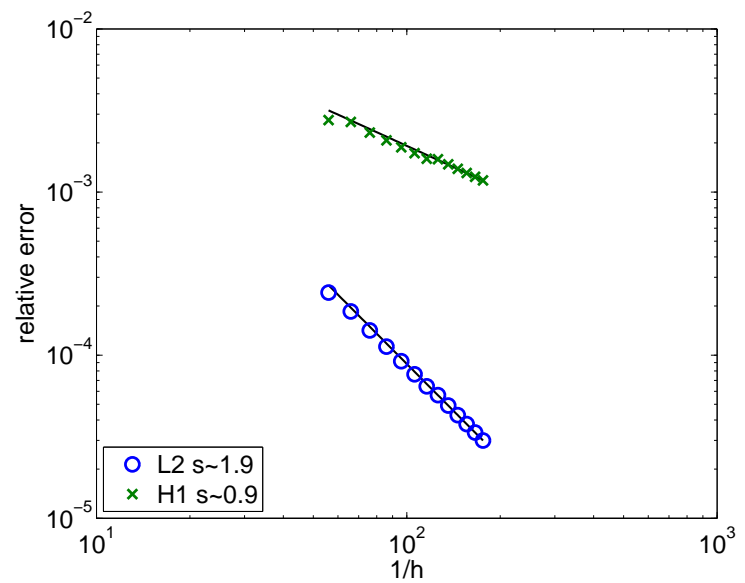

Figure 8. Error in relative $L^{2}$ and $H^{1}$ norms for the Mode I crack problem

\section{FAST SOLVER USING THE FAST FOURIER TRANSFORM}

As discussed in Section 6.1, the main disadvantage of the DPM is that it requires $4|\omega|$ solutions of the auxiliary problem in order to construct the BEP (41). Here we follow the formulation for a fast solution method given by [21] for the solution of a finite difference formulation and apply an adjusted scheme derived for the FEM used in this paper. This FFT formulation is used to solve the 
regular part of the problem, while the smaller enriched part of the problem must be solved using conventional methods. Therefore a two-step solution is introduced. For a derivation of the FFT formulation used for the solution of the regular, unenriched part of the problem, see Appendix A.

The FFT is used for the fast inversion of the difference operator $\mathbf{L}_{M^{0} N^{0}}$ while requiring only a partial construction of the stiffness matrix for the enriched problem. The method takes advantage of the regularity of the auxiliary problem in addition to the flexibility allowed in choosing its boundary conditions. The boundary conditions of the auxiliary problem need only ensure that the auxiliary problem is well defined. A discussion of the effectiveness of this algorithm for enriched problems is given in Section 8.1.1.

\subsection{Enriched formulation}

Now let us consider the enriched auxiliary problem (13) for $u_{N^{0}}^{*} \in U_{N^{0}}^{*}$. This problem requires additional consideration due to the enriched DOFs within the stiffness matrix.

Let us rewrite the enriched auxiliary problem (13) as follows:

$$
\mathbf{K} u^{e}=f^{e},
$$

where $\mathbf{K}$ is the fully defined stiffness matrix of the auxiliary problem (13) such that for two dimensional problems $\mathbf{K}$ is a $2\left(\left|N^{0}\right|+\left|I_{F H}\right|\right) \times 2\left(\left|N^{0}\right|+\left|I_{F H}\right|\right)$ matrix which contains the operator $\mathbf{L}_{M^{0} N^{0}}$ in addition to the well defined boundary conditions. We do this by simply adding rows containing the boundary equations to $\mathbf{L}_{M^{0} N^{0}}$ at the appropriate locations such that they correspond to the boundary displacements within $u^{e}$. Here $2\left(\left|N^{0}\right|+\left|I_{F H}\right|\right)$ is the total number of DOFs in the system which we will denote $n^{*} . f^{e}$ is an extension of the force vector, $f_{M^{0}}$, in order to include the boundary conditions. As the boundary conditions are homogeneous, this term is extended by 0 to a length of $n^{*}$. The displacement vector remains unchanged, i.e. $u^{e} \equiv u_{N^{0}}^{*} \in U_{N^{0}}^{*}$. The boundary conditions applied to this enriched auxiliary problem are to be the same as for the regular auxiliary problem (49). The auxiliary problem (45) can be structured as follows:

$$
\left[\begin{array}{ll}
\mathbf{K}_{a a} & \mathbf{K}_{a c} \\
\mathbf{K}_{c a} & \mathbf{K}_{c c}
\end{array}\right]\left\{\begin{array}{l}
a \\
c
\end{array}\right\}=\left\{\begin{array}{l}
f^{a} \\
f^{c}
\end{array}\right\},
$$

where $\mathbf{K}_{a a}$ contains all regular equations related to the regular coefficients $a_{n}$ in (6), $\mathbf{K}_{c c}$ contains enriched equations only related to the $c_{n}$ coefficients in (6), while $\mathbf{K}_{a c}$ and $\mathbf{K}_{c a}$ are coupling terms between the regular and enriched FEM. As $\mathbf{K}$ is sparse, each submatrix is also sparse. Here $a$ and $c$ are vectors of the regular terms, $a_{n}$ for $n \in N^{0}$, and enriched terms, $c_{n}$ for $n \in I_{F H}$, respectively, while $f^{a}$ and $f^{c}$ are vectors of the corresponding force terms.

Now we will solve the restated enriched auxiliary problem (45) in two stages:

$$
\begin{gathered}
\left(\mathbf{K}_{c c}-\mathbf{K}_{c a} \mathbf{K}_{a a}^{-1} \mathbf{K}_{a c}\right) c=f^{c}-\mathbf{K}_{c a} \mathbf{K}_{a a}^{-1} f^{a} \\
\mathbf{K}_{a a} a=f^{a}-\mathbf{K}_{a c} c .
\end{gathered}
$$

This two-stage solution method allows us to take advantage of the fast FFT solver to invert $\mathbf{K}_{a a}$. As generally the enriched area of a problem is small in comparison to the global domain area, the matrix $\mathbf{K}_{a a}$, of dimensions $2\left|N^{0}\right| \times 2\left|N^{0}\right|$, is much larger than $\mathbf{K}_{b b}$, of dimensions $2\left|I_{F H}\right| \times 2\left|I_{F H}\right|$. 
In order to solve the first system (47) we require $n^{c}=2\left|I_{F H}\right|$ solutions of the fast solver to calculate the term $\mathbf{K}_{a a}^{-1} \mathbf{K}_{a c}$ in addition to one solution on the right hand side of (47). We are then left with a full matrix system of dimension $n^{c} \times n^{c}$ which can be solved using conventional methods such as LU decomposition. Although the matrix is full, it is small in comparison to the total number of DOFs and therefore the LU matrices do not require a large amount of memory. We then require one additional solution of the fast solver in order to solve (48).

8.1.1. Computational cost Let us denote $n^{a}=2\left|N^{0}\right|$ as the total number of regular DOFs. Let us assume we use the LU decomposition method in order to solve the smaller enriched system (47). Let us denote the matrix system on the left hand side of (47) as $\mathbf{K}_{c c}^{\prime}=\mathbf{K}_{c c}-\mathbf{K}_{c a} \mathbf{K}_{a a}^{-1} \mathbf{K}_{a c}$. Before solving we calculate and store $\mathbf{K}_{c c}^{\prime}$ at a cost of $O\left(n^{c} n^{a} \ln \left(n^{a}\right)+\left(n^{c}\right)^{2}\right)$. Here $O\left(n^{a} \ln \left(n^{a}\right)\right)$ is the number of operations required to solve the regular part of the auxiliary problem once using the FFT formulation (see Appendix A). This must be done $O\left(n^{c}\right)$ number of times to obtain $\mathbf{K}_{a a}^{-1} \mathbf{K}_{a c}$. We then also require $O\left(\left(n^{c}\right)^{2}\right)$ number of operations in order to calculate $\mathbf{K}_{c a} \mathbf{K}_{a a}^{-1} \mathbf{K}_{a c}$. $\mathbf{K}_{c c}^{\prime}$ is a full matrix, therefore its bandwidth is $b^{c}=n^{c}$ and the LU decomposition of $\mathbf{K}_{c c}^{\prime}$ then requires $O\left(n^{c}\left(b^{c}\right)^{2}\right)=O\left(\left(n^{c}\right)^{3}\right)$ operations [27]. When geometrical enrichment is used, the ratio between the number of enriched DOFs, $n^{c}$, and the number of regular DOFs, $n^{a}$, approaches a constant. For the following discussion we assume that a geometrical enrichment is used. In the case of large problems we have $\left(n^{c}\right)^{3}>>n^{c} n^{a} \ln \left(n^{a}\right)+\left(n^{c}\right)^{2}$, and therefore an approximation of the number of operations needed for the preparation of this method for solution is of order $O\left(\left(n^{c}\right)^{3}\right)$. The total cost of then solving the enriched auxiliary problem is $O\left(n^{c} b^{c}\right)=O\left(\left(n^{c}\right)^{2}\right)$ for the solution of (47) through the forward and backward substitution of the LU matrices, and $O\left(n^{a} \ln \left(n^{a}\right)\right)$ for the solution of (48). For large problems, we have $\left(n^{c}\right)^{2}>>n^{a} \ln \left(n^{a}\right)$ and therefore the order of operations for the solution of the auxiliary problem becomes $O\left(\left(n^{c}\right)^{2}\right)$. However, as discussed in 6.1 , the DPM requires $4|\omega|+2$ solutions of the auxiliary problem. Therefore we have a total number of required operations at $O\left(\left(n^{c}\right)^{3}+(4|\omega|+2)\left(n^{c}\right)^{2}\right)$. Using appropriately chosen basis functions should restrict the required number of basis functions, $|\omega|$, such that $|\omega|<<n^{c}$, for example see [15]. Here we have neglected the cost of solving the BEP (39) as due to its small size the cost of its solution is small in comparison to the solution of the auxiliary problem.

Table I shows the computational cost of the DPM presented here in comparison to the classical FEM method using LU decomposition for the BVP. As we expect $n^{c}<<n^{a}$ and $|\omega|$ to be independent of mesh refinement, the FTT formulation compares well with the LU decomposition. However, for very large problems using geometrical enrichment, $n^{c}$ could become sufficiently large such that the method is no longer quicker to solve. However, the DPM holds two significant advantages over classical methods regardless of the size of the problem. The regular grid is advantageous at the meshing stage as there is no need to construct a mesh which conforms to the domain geometry. Further to this, its regularity means that the stiffness matrix can be defined explicitly, e.g. using a finite difference scheme. Therefore, the DPM allows us to avoid two expensive steps in the preprocessing stage.

In order to put the numbers shown in Table $\mathrm{I}$ in context, let us consider a problem with an area ratio of $A_{F} / A=1 / 400$. This is equivalent to a square $1 \mathrm{~m} \times 1 \mathrm{~m}$ domain with an enrichment area at a crack tip of dimension $0.05 \mathrm{~m} \times 0.05 \mathrm{~m}$. Now we know that, for large problems, $n^{c} / n^{a} \approx\left(A_{F} / A\right)$ and therefore have $n^{c} \approx\left(A_{F} / A\right) n^{a}$. Therefore we have the order of 
Table I. Comparison of DPM with classical FEM

\begin{tabular}{l|c|c} 
& FEM with LU decomposition & DPM with FFT \\
\hline Mesh generation & $\begin{array}{c}\text { Must conform } \\
\text { to boundaries }\end{array}$ & Regular \\
\hline Build K & Gauss quadrature & Define explicitly \\
\hline $\begin{array}{c}\text { Cost of matrix } \\
\text { decomposition }\end{array}$ & $O\left(\left(n^{*}\right)^{2}\right)$ & $O\left(\left(n^{c}\right)^{3}\right)$ \\
\hline $\begin{array}{c}\text { Cost of solution } \\
\text { solutions }\end{array}$ & $O\left(\left(n^{*}\right)^{3 / 2}\right)$ & $(4|\omega|+2) O\left(\left(n^{c}\right)^{2}\right)$ \\
\hline $\begin{array}{c}\text { Cost of additional } \\
\text { solution }\end{array}$ & $O\left(\left(n^{*}\right)^{3 / 2}\right)$ & $O\left(\left(n^{c}\right)^{2}\right)$
\end{tabular}

operations required for the construction and solution of the auxiliary problem as $O\left(\left(\left(A_{F} / A\right) n^{a}\right)^{3}\right)$ and $O\left((4|\omega|+2)\left(\left(A_{F} / A\right) n^{a}\right)^{2}\right)$ respectively, which in this case equates to $O\left(10^{-8}\left(n^{a}\right)^{3}\right)$ and $O\left(10^{-6}(4|\omega|+2)\left(n^{a}\right)^{2}\right)$. Now in order for the cost of matrix construction for the DPM to be less than that for the LU decomposition we require $O\left(10^{-8}\left(n^{a}\right)^{3}\right)<O\left(\left(n^{*}\right)^{2}\right)$. If we approximate $n^{*} \approx$ $n^{a}$, this leads to the condition $n^{a}<O\left(10^{8}\right)$. Similarly, in order for the cost for the solution for DPM to be less than that for the LU decomposition we require $O\left(10^{-6}(4|\omega|+2)\left(n^{a}\right)^{2}\right)<O\left(\left(n^{*}\right)^{3 / 2}\right)$. Rearranging and applying the same approximation, we have $n^{a}<O\left(10^{12} /(4|\omega|+2)^{2}\right)$, where we expect $|\omega|<<n^{a}$. For $n^{a}$ in the region of these values the DPM with the FFT algorithm is expected to become more expensive than the classical FEM when ignoring the added cost of Gauss quadrature and mesh generation. While a study is required in order to establish the true effectiveness of this formulation, the discussion provided here demonstrates the potential of the method.

Finally, we note the final row of Table I. Here we provide the order of operations required for the solution of any additional problems using the same geometry but different boundary conditions or force terms. In this case the BEP can be stored from the first problem and reused, therefore avoiding the need for $4|\omega|$ solutions of the auxiliary problem.

\section{DISCUSSION}

Here the DPM formulation has been significantly enhanced compared to that presented in [12]. The reduction of the problem to a system of basis functions along the crack tip greatly increases the efficiency of the solution method. The number of solutions of the auxiliary problem needed is reduced from $2|\gamma|$ to $4|\omega|$. $\gamma$ increases with grid refinement $\left(|\gamma| \propto \sqrt{n^{0}}\right)$, however $\omega$ can be independent of grid refinement. Therefore the greater the grid refinement, the greater the efficiency of the new methodology compared to that presented in [12].

In this paper, local splines are used as basis functions along the entire physical boundary. Second order, local splines were chosen in anticipation of an error at the crack tip due to the gradient singularity. However, it was found that the density along the crack boundary can be a smooth function. Therefore higher order or non-local functions could be used as basis functions along the outer boundary and along the crack boundary. In doing so one would expect that on fine grids considerably fewer boundary interpolation functions than boundary grid nodes will be needed for achieving a comparable accuracy. This would then reduce the number of basis functions, and therefore auxiliary problem solutions, needed to achieve a desired accuracy, further improving the 
efficiency of the method. While this has been achieved before for regular problems, this is the first time it has been applied to crack problems with a singularity on the boundary.

Further to this, the new methodology allows for an easier and more flexible application of boundary conditions. Whereas with the previous method, the boundary conditions were approximated on the grid nodes, here the boundary conditions can be implemented to any accuracy on the physical boundary.

A fast solution method is suggested in Section 8. Such methods could allow the DPM to be an efficient solution method for crack propagation problems. For such problems the regular part of the stiffness matrix, $\mathbf{K}_{a a}$, would remain unchanged as the crack propagates. Therefore only the enriched terms, $\mathbf{K}_{a c}, \mathbf{K}_{c a}$ and $\mathbf{K}_{c c}$, would require recalculation at each iteration of the crack length. As a regular grid is used and therefore the difference equations are known throughout (50), (51), these matrices can be constructed explicitly at relatively low computational cost. The quick solver for the DPM could then provide a quick solution method at each iteration leading to a potentially efficient DPM solution method for crack propagation problems.

\section{CONCLUSION}

Within this paper the successful reduction of the DPM to the physical boundary has been demonstrated for LEFM problems containing a discontinuity across the crack and singular derivatives at the crack tip. It has been shown that by enriching the problem near the crack with a discontinuous function with singular derivatives, the trace needed at the crack boundary can be reduced to the regular function and its normal derivative only. It is demonstrated that the trace along the crack boundary can be reduced to a regular function if the singularity is introduced within the FEM space. The formulation requires much fewer solutions of the auxiliary problem than previous formulations for the solution of LEFM problems such as in [12] and therefore offers significant gains in efficiency. Near optimal convergence rates are achieved for the test cases solved, of 1.9 for the $L^{2}$ norm and 0.9 for the $H^{1}$ norm. A fast solution method using the FFT is suggested which takes advantage of the DPM's ability to solve complex problems on a regular, rectangular grid potentially leading to further gains in efficiency. The efficiency of the method will be demonstrated through numerical tests to be presented in a future paper.

\section{ACKNOWLEDGEMENT}

This work is supported by the U.K. Engineering and Physical Sciences Research Council (EPSRC) under DTA award and EDF.

\section{A. FINITE FOURIER TRANSFORM FORMULATION}

Here will will follow the formulation of [21] for a finite difference scheme to derive the FFT formulation for our FEM on a regular, square grid. Consider the auxiliary problem (13) under the 
constraint $u_{N^{0}}^{*} \in U_{N^{0}}$ such that the displacement $u_{N^{0}}^{*}=u_{N^{0}}$, i.e. is a regular, unenriched function. The method involves extending our auxiliary problem of dimension $\left|N^{0}\right|$ to a larger problem of dimension $4\left|N^{0}\right|$. This larger problem is in fact solved with periodic boundary conditions, however the asymmetric nature of the extension results in a unique solution and mixed boundary conditions on our original $\left|N^{0}\right|$ problem [21]. Let us denote the extended grid sets $M^{e}$ as all the plane nodes and $N^{e}$ as all nodes (plane and hollow) in Figure 9. The original auxiliary domain is shown in gray. Considering this problem only, the plane nodes denote the $M^{0}$ grid set while the hollow nodes denote the nodes on the boundary belonging to $N^{0}$. The extended problem will be solved such that mixed boundary conditions are applied on these hollow nodes.

Let us denote $\tilde{N}^{e}=\sqrt{\left|N^{e}\right|}$ as the number of rows and columns of nodes in the extended problem and $\tilde{N}^{0}=\sqrt{\left|N^{0}\right|}$ as the number of rows and columns of nodes in the original problem, here $\tilde{N}^{0}=\tilde{N}^{e} / 2$. We extend the force term $f_{M^{0}}=\left(f_{M^{0}}^{(1)}, f_{M^{0}}^{(2)}\right)$ asymmetrically onto the entire $N^{e}$ domain as follows:

$f_{m_{1}, m_{2}}=\left(f_{m_{1}, m_{2}}^{(1)}, f_{m_{1}, m_{2}}^{(2)}\right) \begin{cases}=\left(f_{m_{1}, m_{2}}^{(1)}, f_{m_{1}, m_{2}}^{(2)}\right) & \text { if } m_{1}, m_{2} \leq \tilde{N}^{0}, \\ =\left(-f_{\tilde{N}^{e}-m_{1}, m_{2}}^{(1)}, f_{\tilde{N}^{e}-m_{1}, m_{2}}^{(2)}\right) & \text { if } m_{1}>\tilde{N}^{0}, m_{2} \leq \tilde{N}^{0}, \\ =\left(f_{m_{1}, \tilde{N}^{e}-m_{2}}^{(1)},-f_{m_{1}, \tilde{N}^{e}-m_{2}}^{(2)}\right) & \text { if } m_{1} \leq \tilde{N}^{0}, m_{2}>\tilde{N}^{0}, \\ =\left(-f_{\tilde{N}^{e}-m_{1}, \tilde{N}^{e}-m_{2}}^{(1)},-f_{\tilde{N}^{0}-m_{1}, \tilde{N}^{0}-m_{2}}^{(2)}\right) & \text { if } m_{1}>\tilde{N}^{0}, m_{2}>\tilde{N}^{0},\end{cases}$

where here $m_{1}, m_{2}=1,2, . ., \tilde{N}^{e}$ and $m_{1}=m_{2}=1$ is chosen to be the point at the top left hand corner of the domain. This extension is shown symbolically in Figure 9. Here the reflection of the letters represent a reflection of the field. The force term is defined as zero on the hollow nodes. The extension is defined such that the sum of the force terms over the entire extended domain are zero, i.e. $\sum_{n=1}^{\left|N^{e}\right|} f_{n}^{(j)}=0$ for $j=(1,2)$. This restricts the possibility for rigid body motion that is otherwise present for problems with periodic boundary conditions and therefore ensures a unique solution to the problem.

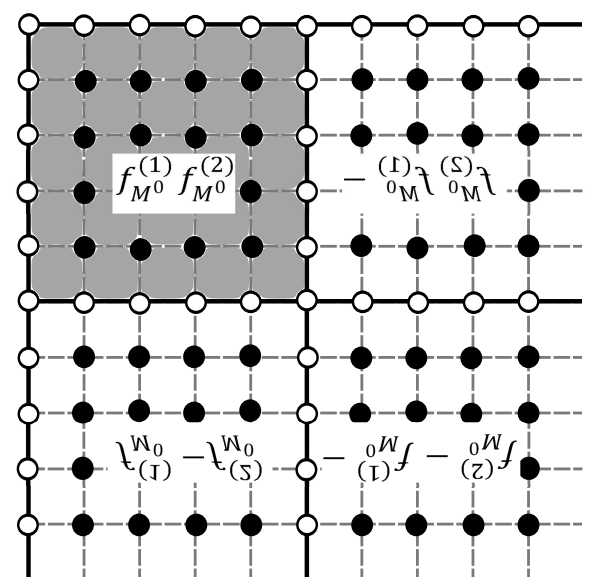

Figure 9. Extended domain for the FFT formualtion. Plane nodes denote $M^{0}$ nodes and hollow nodes denote nodes upon which the boundary conditions exist 
This formulation enforces the following boundary conditions for the solution $u_{N^{e}}(x, y)=$ $\left(u_{N^{e}}^{(1)}(x, y), u_{N^{e}}^{(2)}(x, y)\right)$ on the hollow nodes shown in Figure 9:

$$
\begin{gathered}
u_{N^{e}}^{(1)}(0, y)=u_{N^{e}}^{(1)}\left(\tilde{N}^{e} h / 2, y\right)=0, \\
\frac{\partial u_{N^{e}}^{(2)}(0, y)}{\partial x}=\frac{\partial u_{N^{e}}^{(2)}\left(\tilde{N}^{e} h / 2, y\right)}{\partial x}=0, \\
u_{N^{e}}^{(2)}(x, 0)=u_{N^{e}}^{(2)}\left(x, \tilde{N}^{e} h / 2\right)=0, \\
\frac{\partial u_{N^{e}}^{(1)}(x, 0)}{\partial y}=\frac{\partial u_{N^{e}}^{(1)}\left(x, \tilde{N}^{e} h / 2\right)}{\partial y}=0,
\end{gathered}
$$

Discretizing the elastostatic equation (1) using a FEM formulation such as that given in Section 3.2 for an unenriched displacement field, $u_{N^{0}} \in U_{N^{0}}$, leads to the following equations for node $n, j=m_{1}, m_{2}$ :

$$
\begin{gathered}
\frac{(\lambda+2 \mu)}{6} A_{n, j}^{(1)}+\frac{\mu}{6} B_{n, j}^{(1)}+\frac{(\lambda+\mu)}{2} C_{n, j}^{(2)}=\tilde{f}_{n, j}^{(1)}, \\
\frac{\mu}{6} A_{n, j}^{(2)}+\frac{(\lambda+2 \mu)}{6} B_{n, j}^{(2)}+\frac{(\lambda+\mu)}{2} C_{n, j}^{(1)}=\tilde{f}_{n, j}^{(2)},
\end{gathered}
$$

where $A_{n, j}^{(i)}, B_{n, j}^{(i)}$ and $C_{n, j}^{(i)}$ represent the following difference equations:

$$
\begin{gathered}
A_{n, j}^{(i)}=-u_{n-1, j-1}^{(i)}-4 u_{n-1, j}^{(i)}-u_{n-1, j+1}^{(i)}+2 u_{n, j-1}^{(i)}+8 u_{n, j}^{(i)}+ \\
2 u_{n, j+1}^{(i)}-u_{n+1, j-1}^{(i)}-4 u_{n+1, j}^{(i)}-u_{n+1, j+1}^{(i)}, \\
B_{n, j}^{(i)}=-u_{n-1, j-1}^{(i)}+2 u_{n-1, j}^{(i)}-u_{n-1, j+1}^{(i)}-4 u_{n, j-1}^{(i)}+8 u_{n, j}^{(i)}- \\
4 u_{n, j+1}^{(i)}-u_{n+1, j-1}^{(i)}+2 u_{n+1, j}^{(i)}-u_{n+1, j+1}^{(i)}, \\
C_{n, j}^{(i)}=-u_{n-1, j-1}^{(i)}+u_{n-1, j+1}^{(i)}+u_{n+1, j-1}^{(i)}-u_{n+1, j+1}^{(i)},
\end{gathered}
$$

and the force term $\tilde{f}_{n, j}^{(i)}$ is defined as:

$$
\begin{aligned}
& \tilde{f}_{n, j}^{(i)}=\frac{h^{2}}{36}\left(f_{n-1, j-1}^{(i)}+4 f_{n-1, j}^{(i)}+f_{n-1, j+1}^{(i)}+\right. 4 f_{n, j-1}^{(i)}+16 f_{n, j}^{(i)}+ \\
&\left.4 f_{n, j+1}^{(i)}+f_{n+1, j-1}^{(i)}+4 f_{n+1, j}^{(i)}+f_{n+1, j+1}^{(i)}\right) .
\end{aligned}
$$

Equations (50) and (51) are the $\mathrm{x}$ and $\mathrm{y}$ component equations within the stiffness matrix belonging to node $n, j$. As our problem is homogeneous, this equation is the same throughout the stiffness matrix.

Now we introduce the following discrete Fourier transform (DFT) in the first variable, where here $i=\sqrt{-1}$,

$$
U_{k, j}^{(j)}=\sum_{n=1}^{\tilde{N}^{e}} u_{n, j}^{(j)} \exp \left(\frac{-2 \pi i(k-1)(n-1)}{\tilde{N}^{e}}\right), \quad 1 \leq k \leq \tilde{N}^{e},
$$

and the inverse DFT, 


$$
u_{n, j}^{(j)}=\frac{1}{\tilde{N}^{e}} \sum_{k=1}^{\tilde{N}^{e}} U_{k, j}^{(j)} \exp \left(\frac{2 \pi i(k-1)(n-1)}{\tilde{N}^{e}}\right), \quad 1 \leq n \leq \tilde{N}^{e} .
$$

We use the same notation for the force term, such that the DFT is given by:

$$
F_{k, j}^{(j)}=\sum_{n=1}^{\tilde{N}^{e}} \tilde{f}_{n, j}^{(j)} \exp \left(\frac{-2 \pi i(k-1)(n-1)}{\tilde{N}^{e}}\right), \quad 1 \leq k \leq \tilde{N}^{e} .
$$

We note here that the DFT is applied to $\tilde{f}_{n, j}^{(j)}$, defined in (52), rather than $f_{n, j}^{(j)}$. It is possible to transform $f_{n, j}^{(j)}$, however as $\tilde{f}_{n, j}^{(j)}$ is easily calculated the formulation is simplified slightly by using $\tilde{f}_{n, j}^{(j)}$ here.

The DFT in both variables is then given by:

$$
\bar{U}_{k, m}^{(j)}=\sum_{j=1}^{\tilde{N}^{e}} U_{k, j}^{(j)} \exp \left(\frac{-2 \pi i(m-1)(j-1)}{\tilde{N}^{e}}\right), \quad 1 \leq m \leq \tilde{N}^{e},
$$

with the corresponding inverse DFT:

$$
U_{k, j}^{(j)}=\frac{1}{\tilde{N}^{e}} \sum_{m=1}^{\tilde{N}^{e}} \bar{U}_{k, m}^{(j)} \exp \left(\frac{2 \pi i(m-1)(j-1)}{\tilde{N}^{e}}\right), \quad 1 \leq j \leq \tilde{N}^{e},
$$

where again equivalent expressions exist for the force terms.

Substituting the inverse DFTs (54) and then (57) into the difference equations (50), (51) and rearranging we arrive at the following equations:

$$
\begin{aligned}
& a_{k, m} \bar{U}_{k, m}^{(1)}+b_{k, m} \bar{U}_{k, m}^{(2)}=\bar{F}_{k, m}^{(1)}, \\
& d_{k, m} \bar{U}_{k, m}^{(2)}+b_{k, m} \bar{U}_{k, m}^{(1)}=\bar{F}_{k, m}^{(2)},
\end{aligned}
$$

where the terms $a_{k, m}, b_{k, m}$ and $c_{k, m}$ are found to be:

$$
\begin{gathered}
a_{k, m}=\frac{(\lambda+3 \mu)}{6}\left[8-4 \cos \left(\frac{2 \pi(m-1)}{\tilde{N}^{e}}\right) \cos \left(\frac{2 \pi(k-1)}{\tilde{N}^{e}}\right)\right] \\
\quad-\frac{(4 \lambda+6 \mu)}{3} \cos \left(\frac{2 \pi(k-1)}{\tilde{N}^{e}}\right)+\frac{2 \lambda}{3} \cos \left(\frac{2 \pi(m-1)}{\tilde{N}^{e}}\right), \\
b_{k, m}=2(\lambda+\mu) \sin \left(\frac{2 \pi(m-1)}{\tilde{N}^{e}}\right) \sin \left(\frac{2 \pi(k-1)}{\tilde{N}^{e}}\right), \\
d_{k, m}=\frac{(\lambda+3 \mu)}{6}\left[8-4 \cos \left(\frac{2 \pi(m-1)}{\tilde{N}^{e}}\right) \cos \left(\frac{2 \pi(k-1)}{\tilde{N}^{e}}\right)\right] \\
\quad-\frac{(4 \lambda+6 \mu)}{3} \cos \left(\frac{2 \pi(m-1)}{\tilde{N}^{e}}\right)+\frac{2 \lambda}{3} \cos \left(\frac{2 \pi(k-1)}{\tilde{N}^{e}}\right) .
\end{gathered}
$$

Equations (58) can be inverted for $1 \leq k \leq \tilde{N}^{e}, 1 \leq m \leq \tilde{N}^{e}$ and $k m>1$ such that: 


$$
\begin{aligned}
& \bar{U}_{k, m}^{(1)}=\frac{d_{k, m} \bar{F}_{k, m}^{(1)}-b_{k, m} \bar{F}_{k, m}^{(2)}}{a_{k, m} d_{k, m}-b_{k, m}^{2}}, \\
& \bar{U}_{k, m}^{(2)}=\frac{a_{k, m} \bar{F}_{k, m}^{(2)}-b_{k, m} \bar{F}_{k, m}^{(1)}}{a_{k, m} d_{k, m}-b_{k, m}^{2}} .
\end{aligned}
$$

For $k=m=1$ we have $a_{1,1}=b_{1,1}=d_{1,1}=0$ and therefore from equations (58) we have $\bar{F}_{1,1}^{(1)}=\bar{F}_{1,1}^{(2)}=0$. In this case equations (58) are satisfied for any displacement terms and therefore $\bar{U}_{1,1}^{(1)}, \bar{U}_{1,1}^{(2)}$ are not unique. We therefore set $\bar{U}_{1,1}^{(1)}=\bar{U}_{1,1}^{(2)}=0$ in order to ensure a unique solution is obtained.

For this regular problem, let us denote $n^{e}=2\left|N^{e}\right|$ as the total number of DOFs within our extended problem. The above formulation allows the solution to the regular auxiliary problem (13) under the constraint $u_{N^{0}}^{*}=u_{N^{0}} \in U_{N^{0}}$ to be solved in $O\left(n^{e} \ln \left(n^{e}\right)\right)$ operations [21]. In relation to the dimensions of the original auxiliary problem, as $n^{e}=4 n^{0}$, this equates to $O\left(4 n^{a} \ln \left(4 n^{a}\right)\right)=$ $O\left(n^{a} \ln \left(n^{a}\right)\right)$.

\section{REFERENCES}

1. Ryaben'kii VS. Method of Difference Potentials and Its Applications. 2002 edn., Springer, 2001.

2. Ryaben'kii VS, Tsynkov SV. A Theoretical Introduction to Numerical Analysis. CRC Press, 2006.

3. Kansa E, Shumlak U, Tsynkov S. Discrete Calderon's projections on parallelepipeds and their application to computing exterior magnetic fields for FRC plasmas. Journal of Computational Physics Feb 2013; 234:172-198, doi:10.1016/j.jcp.2012.09.033.

4. Lim H, Utyuzhnikov SV, Lam YW, Turan A, Avis MR, Ryaben'kii VS, Tsynkov SV. Experimental validation of the active noise control methodology based on difference potentials. AIAA Journal Apr 2009; 47(4):874-884, doi: $10.2514 / 1.32496$.

5. Loncaric J, Ryaben'kii VS, Tsynkov SV. Active shielding and control of noise. SIAM Journal on Applied Mathematics Jan 2001; 62(2):563-596, doi:10.1137/S0036139900367589.

6. Ryaben'kii VS, Utyuzhnikov SV. Active shielding model for hyperbolic equations. IMA Journal of Applied Mathematics Dec 2006; 71(6):924-939, doi:10.1093/imamat/hx1015.

7. Aliabadi MH, Rooke DP, Cartwright DJ. An improved boundary element formulation for calculating stress intensity factors: Application to aerospace structures. The Journal of Strain Analysis for Engineering Design 1987; 22(4):203-207, doi:10.1243/03093247V224203.

8. Blandford GE, Ingraffea AR, Liggett JA. Two-dimensional stress intensity factor computations using the boundary element method. International Journal for Numerical Methods in Engineering 1981; 17(3):387-404, doi:10.1002/ nme.1620170308.

9. Portela A, Aliabadi M, Rooke D. Dual boundary element method. effective implementation for crack problems. International Journal for Numerical Methods in Engineering 1992; 33(6):1269-1287.

10. Telles J, Guimarães S. Green's function: a numerical generation for fracture mechanics problems via boundary elements. Computer Methods in Applied Mechanics and Engineering Aug 2000; 188(4):847-858, doi:10.1016/ S0045-7825(99)00366-7.

11. Weaver J. Three-dimensional crack analysis. International Journal of Solids and Structures 1977; 13(4):321-330, doi:10.1016/0020-7683(77)90016-6.

12. Woodward WH, Utyuzhnikov SV, Massin P. On the application of the method of difference potentials to linear elastic fracture mechanics. International Journal for Numerical Methods in Engineering 2015; 103(10):703-736, doi:10.1002/nme.4903.

13. Düster A, Parvizian J, Yang Z, Rank E. The finite cell method for three-dimensional problems of solid mechanics. Computational methods in Applied Mechanics and Engineering 2008; 197(45-48):3768-3782, doi: http://dx.doi.org/10.1016/j.cma.2008.02.036.

14. Ryaben'kii VS, Utyuzhnikov SV. An algorithm of the method of difference potentials for domains with cuts. Journal of Applied Numerical Mathematics 2015; 93:254 - 261, doi:http://dx.doi.org/10.1016/j.apnum.2014.02. 007. 
15. Medvinsky M, Tsynkov S, Turkel E. The method of difference potentials for the Helmholtz equation using compact high order schemes. Journal of Scientific Computing May 2012; 53(1):150-193, doi:10.1007/ s10915-012-9602-y.

16. Kamenetskii DS, Ryabenkii VS. Solution of boundary value problems for the Laplace equation in a domain with a cut by the method of dierence potentials. Preprint 33, Keldysh Institute for Applied Mathematics, USSR Academy of Sciences, Moscow, USSR 1990. [Russian].

17. Kamenetskii DS. A numerical method for solving a singular boundary value problem for the Chaplygin equation in the hodograph plane. Preprint 60, Keldysh Institute for Applied Mathematics, USSR Academy of Sciences, Moscow, USSR 1992. [Russian].

18. Shifrin EG, Kamenetskii DS. Application of the hodograph method to nozzle guide vane profiling. Russian Journal of Computational Mechanics 1993; 3:80-107. [Russian].

19. Magura S, Petropavlovsky S, Tsynkov S, Turkel E, High-order numerical solution of the Helmholtz equation for domains with reentrant corners. Appl. Numer. Math. 2017; 118:87-116, doi:10.1016/j.apnum.2017.02.013.

20. Britt S, Petropavlovsky S, Tsynkov S, Turkel E, Computation of Singular Solutions to the Helmholtz Equation with High Order Accuracy. Appl. Numer. Math. 2015; 93(C):215-241, doi:10.1016/j.apnum.2014.10.006.

21. Wiegmann A. Fast Poisson, Fast Helmholtz and fast linear elastostatic solvers on rectangular parallelepipeds. Lawrence Berkeley National Laboratory 1999, doi:10.2172/982430.

22. Laborde P, Pommier J, Renard Y, Salaün M. High-order extended finite element method for cracked domains. International Journal for Numerical Methods in Engineering 2005; 64(3):354-381, doi:10.1002/nme.1370.

23. Xiao QZ, Karihaloo BL, Direct evaluation of accurate coefficients of the linear elastic crack tip asymptotic field. Fatigue \& Fracture of Engineering Materials \& Structures 2003; 26(8):31460-2695, doi:10.1046/j.1460-2695. 2003.00648.x.

24. Ryaben'kii VS, Local splines. Computer Methods in Applied Mechanics and Engineering 1975; 5(2):211-225, doi:10.1016/0045-7825(75)90053-5

25. Zhang L, Li L. On superconvergence of isoparametric bilinear finite elements. Communications in Numerical Methods in Engineering 1996; 12(12):849-862, doi:10.1002/(SICI)1099-0887(199612)12:12/849:: AID-CNM25 3.0.CO;2-N.

26. Jovanovic BS. Finite difference schemes for boundary value problems with generalized solutions. Journal of Mathematics, Novi Sad 2000; 30(3):47-58.

27. Bathe KJ. Finite Element Procedures. Klaus-Jurgen Bathe, 2006. 